\title{
New Developments in Deformation Experiments at High Pressure
}

\author{
William B. Durham \\ University of California, \\ Lawrence Livermore National Laboratory, P.O. Box 808 \\ Livermore, California 94550 \\ Donald J. Weidner \\ Department?? \\ State University of New York \\ Stony Brook, New York ZIP?? \\ Shun-ichiro Karato \\ Departmentof Geology and Geophysics \\ 319 Kline Geology Laboratory, Yale University \\ New Haven, Connecticut 06520 \\ Yanbin Wang \\ GeoSoilEnviroCARS \\ University of Chicago \\ Chicago, Illinois ZIP??
}

\section{INTRODUCTION}

Although the importance of rheological properties in controlling the dynamics and evolution of the whole mantle of Earth is well-recognized, experimental studies of rheological properties and deformation-induced microstructures have mostly been limited to low-pressure conditions. This is mainly a result of technical limitations in conducting quantitative rheological experiments under high-pressure conditions. A combination of factors is changing this situation. Increased resolution of composition and configuration of Earth's interior has created a greater demand for well-resolved laboratory measurement of the effects of pressure on the behavior of materials. Higher-strength materials have become readily available for containing high-pressure research devices, and new analytical capabilities - in particular very bright synchrotron X-ray sources - are now readily available to high-pressure researchers.

One of the biggest issues in global geodynamics is the style of mantle convection and the nature of chemical differentiation associated with convectional mass transport. Although evidence for deep mantle circulation has recently been found through seismic tomography (e.g., van der Hilst et al. (1997)), complications in convection style have also been noted. They include (1) significant modifications of flow geometry across the mantle transition zone as seen from high resolution tomographic studies (Fukao et al. 1992; Masters et al. 2000; van der Hilst et al. 1991) and (2) complicated patterns of flow in the deep lower mantle $(\sim 1500-2500 \mathrm{~km})$, perhaps caused by chemical heterogeneity (Kellogg et al. 1999; van der Hilst and Karason 1999).

These studies indicate that while large-scale circulation involving the whole mantle no doubt occurs, significant deviations from simple flow geometry are also present. Two mineral properties have strong influence on convection: (1) density and (2) viscosity (rheology) contrasts. In the past, the effects of density contrast have been emphasized 
(Honda et al. 1993; Kellogg et al. 1999; Tackley et al. 1993), and the influence of rheology has been demonstrated by geodynamic modeling (Davies 1995; Karato et al. 2001).

Rheological properties of the Earth's mantle can be inferred from analyses of geodynamic data such as post-glacial crustal movement and gravity anomalies (Mitrovioca and Forte 1997; Peltier 1998). While such geodynamic inference provides an important data set on rheological structures of the Earth, there are serious limitations for these approaches. First, the resolution of post-glacial rebound data to infer mantle viscosity is limited to $\sim 1200 \mathrm{~km}$. Below that depth, there is little constraint for viscosity from post-glacial rebound (Mitrovioca and Peltier 1991). Furthermore, strain magnitude involved in post-glacial rebound is much smaller than strain magnitude involved in convection, and this raises the issue of effects of transient rheology (Karato 1998). Gravity data (e.g., the geoid) have better sensitivity to radial variation in viscosity in the deep mantle (Hager 1984), but they suffer from non-uniqueness (King 1995; Thoraval and Richards 1997). Therefore, experimental studies on mineral rheology remain a vital component in inferring mantle rheology.

High-pressure rheology may also hold some of the keys for understanding deep earthquakes. Four classes of mechanism have been suggested over the years, including thermal runaway instabilities (Hobbs and Ord 1988; Ogawa 1987), instabilities accompanying recrystallization (Post 1977), dehydration embrittlement (Jiao et al. 2000; Raleigh and Paterson 1965), and instabilities associated with polymorphic phase transformations (see also reviews by Green and Houston (1995) and Kirby et al. (1996)). Again, the main reason uncertainty still exists is the poor knowledge of materials properties at high pressures. Experimental testing of the thermal runaway involved in plastic instability requires high rates of deformation, which are difficult to obtain in small samples; modeling would be a useful tool for this mechanism, but critical parameters for models (rheological properties of deep Earth materials) have been missing so far (Hobbs and Ord 1988; Karato et al. 2001). Well-controlled deformation experiments under deep mantle conditions are needed to provide greater understanding of the shearing instability associated with the phase transformations of olivine to wadsleyite and ringwoodite (Burnley et al. 1991; Green et al. 1990) and the possibility of faulting associated with dehydration of the dense hydrous magnesium silicates (Green 2001).

\section{Why is pressure important?}

Pressure is one of the most important variables in defining the ductile rheology of mantle minerals. Where the deformation mechanism is fixed, the effect of pressure on creep strength is given by:

$$
\sigma(P) / \sigma(0) \propto \exp \left(P V^{*} / n R T\right)
$$

where $\sigma(P)$ is the creep strength at pressure $P, V^{*}$ is activation volume, $n$ is the stress exponent, $R$ is the gas constant, and $T$ is temperature. For olivine, assuming an activation volume of $V^{*}=15 \mathrm{~cm}^{3} / \mathrm{mol}, T=1600 \mathrm{~K}$ and $n=3, \sigma(10 \mathrm{GPa}) / \sigma(0)=43$. This is to be compared with the effect of water, which will change the creep strength of olivine by a factor of $\sim 10$ from dry to water-saturated (Hirth and Kohlstedt 1996; Karato and Jung 2002 in press) or partial melting that will affect creep strength by no more than a factor of 2 (Kohlstedt and Chopra 1994). However, the pressure effect is small at the low pressures where studies are made, e.g. $\sigma(0.5 \mathrm{GPa}) / \sigma(0)=1.2$. Therefore, high-pressure studies are necessary in order to determine the flow properties in the deep Earth.

Such large effects of pressure on the flow laws can result in changes of dominant flow mechanisms with depth in the Earth. Karato and Wu (1993) suggest that dislocation flow is overtaken by diffusion flow as the creep mechanism for olivine within the upper 
mantle. Their reasoning is based on the differences in the pressure dependence, i.e., in the value of $V^{*}$ in Equation (1), of the two flow laws.

Pressure is an agent for phase transformation within the Earth. These high-pressure phases, with different crystal structures, will also differ in their mechanical properties. Rheology of high-pressure phases needs to be investigated within the stability field of the mineral. Because of equipment limitations, the flow properties of minerals such as ringwoodite or majorite, which set the strength characteristics of the transition zone, remain virtually unknown.

The effects of water can be well characterized only by high-pressure experiments. At low pressures $(P<0.5 \mathrm{GPa})$, the creep strength of olivine decreases with pressure under water-saturated conditions (Mei and Kohlstedt 2000b; Mei and Kohlstedt 2000a). However, at higher pressures $(P>1 \mathrm{GPa})$, the creep strength of olivine increases with pressure even at water-saturated conditions (Karato and Jung 2002 in press). Therefore results that can be extrapolated to infer creep strength (viscosity) at deep portions $(>30 \mathrm{~km})$ of Earth can be obtained only by quantitative experiments at pressures higher than $\sim 1 \mathrm{GPa}$.

\section{Terminology related to strength and deformation.}

Usage sometimes varies among research disciplines, so let us define a few key terms, taking the language of rock mechanics as a basis (e.g., Jaeger and Cook (1976)). It is assumed that the meaning of the stress $\left(\sigma_{i j}\right)$ and $\operatorname{strain}\left(\varepsilon_{i j}\right)$ tensors are understood. Deformation (used already in the title without definition) refers loosely to any process that results in strain. We take positive values of stress and strain to indicate compression, following geologic convention. Pressure, hydrostatic pressure, and mean normal stress are all equivalently the trace $P$ of the stress tensor, namely,

$$
\sigma_{i j}=\sigma_{i j}^{\prime}+P
$$

where $\sigma_{i j}^{\prime}$ is the deviatoric stress tensor, $i, j=1,2,3$, representing orthogonal directions. Confining pressure $P_{c}$ is the hydrostatic stress generated by laboratory devices to simulate geologic overburden. Although $P$ and $P_{c}$ are different when $\sigma_{i j}^{\prime}$ is non-zero, the difference in most geologic settings is small and the distinction is very often ignored. Different terms for the same physical quantity are sometimes used to connote different situations. Strain can be divided into two categories: elastic, the recoverable portion, which owes its existence to the state of non-zero $\sigma_{i j}$, and inelastic, the permanent strain that remains forever after stress has been removed. Classic rock mechanics usage recognizes two categories of deformation that result in inelastic strain: brittle and ductile. Brittle deformation is associated with discontinuities of displacement and sudden loss of strength (a term to be defined shortly) and is generally not volume conservative. Ductile deformation is associated with finite displacement gradients, retention of strength, and volume conservation at scales above the $\mathrm{nm}$. Note that there are important strainproducing phenomena that do not fit well into either category, in particular those that involve polymorphism (phase change) or chemical reaction. They may be ignored for the purposes of this review. The strength of a material (in specific circumstances called the yield strength, ultimate strength, etc.) is the maximum stress the material can support under the stated environmental conditions. Flow and failure are closely related terms for the state that exists the moment that applied stress matches strength for ductile and brittle materials, respectively.

The term plasticity requires special note. It was originally defined for metals as essentially equivalent to the phenomenon of flow, just defined, but was defined in terms of the yield envelope (Hill 1950) without qualification as to ductile or brittle yield. While metallurgists and most rock rheologists have retained this meaning for the term plasticity 
(e.g., Evans and Kohlstedt [1995] and Poirier [1985], many in the computational branches of geology have taken plasticity to mean any yield process, whether volume conservative or not. In this review, we follow tradition and use the terms ductile and plastic interchangeably.

Here we focus exclusively on ductile deformation, so we use the terms rheology (flow of materials under stress), deformation, and flow interchangeably. The rheology of a material is described by a constitutive law of the type

$$
\dot{\varepsilon}_{i j}=A f\left(\sigma_{i j}^{\prime}\right), A=A(P, T, \ldots)
$$

where all relevant state variables are included in the term $A$. Engineering the containment of very high pressures in the laboratory generally requires high symmetry in the sample assembly. As will be seen below, two basic types of high-symmetry strain environments exist: axisymmetric and rotational. A shorthand form of Equation (3) has evolved for each of these geometries and we will use that shorthand here. For axisymmetric deformation (whose two-dimensional analog is called pure shear), the values of the three principal stresses are $\sigma_{1} \neq \sigma_{2}=\sigma_{3}$, and we simplify the strain rate tensor to a scalar $\dot{\varepsilon}$, which we call the strain rate or shortening rate. The deviatoric stress tensor can be characterized by the scalar $\sigma=\sigma_{1}-\sigma_{3}$, which we call the differential stress or simply stress. For rotational deformation, the strain is approximately simple shear, and Equation (3) becomes a scalar relationship between the shear strain rate, $\dot{\gamma}$, and the shear stress, $\tau$.

Despite the apparent simplicity of this language of strength and deformation, one should always keep two things in mind: (1) the terminology is not universal, and (2) the simple language belies the flow of rocks in nature, which can be counted on to be both inhomogeneous and low symmetry.

\section{A BRIEF HISTORY OF HIGH-PRESSURE APPARATUS}

\section{To 5 GPa: Cylindrical devices}

Confining pressure in rock mechanics testing serves several purposes: (1) simulating geologic overburden, (2) activating mechanisms of flow and fracture that depend on both normal and shear components of stress, and (3) suppressing brittle deformation where one is interested in gathering information on purely ductile phenomena. The scientific needs for doing measurements under pressure are illustrated in detail in several other contributions to this volume. These needs have driven the development of experimental pressure systems since the early days of rock mechanics.

The earliest experimental machines for deforming rocks under elevated confining pressure were some version of a piston pushing on a cylindrical rock held fast in a cylindrical container. A solid or fluid medium within the container, or even walls of the container itself, provided confining pressure to the rock sample. The reader may find an early history of such machines in Tullis and Tullis (1986), for example. The direction of apparatus development after 1900 was influenced primarily by P.W. Bridgman, who made such rapid advances in the design of hydrostatic equipment that the only reasonable path for rock mechanics to follow was to adapt deformation to Bridgman's vessels. Bridgman, who conceived the dynamic piston seal still commonly used, was involved in that adaptation (Bridgman 1952c), although he was no longer alone in his efforts. Continuing to this day, virtually every new deformation rig is some adaptation of a hydrostatic design. The backbone of the history of deformation machines is mostly a history of pressure vessels.

The technological breakthrough behind modern gas and liquid pressure vessels was 
the Bridgman unsupported area packing (Bridgman 1914), a geometry that intensified the vessel's own pressure at the point of seal and made it possible to "reach without leak any pressure allowed by the mechanical strength of the walls of the containing vessels" (Bridgman 1952b). Bridgman regularly worked at pressures in excess of $1 \mathrm{GPa}$ and occasionally reached $2 \mathrm{GPa}$ with his fluid vessels (Bridgman 1935b). The application of the Bridgman-seal vessel to rock deformation led to the creation of what is commonly called in rock mechanics the "triaxial" apparatus (which is somewhat inappropriate, because the stress environment in the fluid pressure medium can be no lower than axisymmetric). Griggs, who learned the techniques of high pressure from Bridgman and shared his "gift for gadgets" (Rubey 1972), initiated this application by developing a version of the unsupported area packing around a solid moving piston (Fig. 1), thereby allowing sample shortening to be equated (with appropriate corrections for elasticity) to piston displacement (Griggs 1936). Griggs (1936) added a second innovation to the design, a pressure-compensating double piston that automatically kept pressure constant even as the sample was shortened (Fig. 1). Further development of the triaxial rig was aided by advances such as the controlled-clearance (Newhall) packing to reduce piston friction (Handin 1953; Turner et al. 1956); internal heating to achieve higher sample temperatures (Griggs et al. 1960); and the internal force gauge, which removed piston friction from the measurement of differential force on the sample (Heard and Carter 1968; Paterson 1970). This list is by no means exhaustive. A notable recent development is the proliferation in many of the world's most renown rock mechanics laboratories of one particular triaxial apparatus, the Paterson rig, which is a $0.3-\mathrm{GPa}$ ready-to-use system complete with actuation and internal force gauging for both axial and highdisplacement torsional deformation (Paterson 1990; Paterson and Olgaard 2000). As of this writing, there are ten such vessels in use in North America and Europe.

Practical matters related to sealing a high-pressure fluid have generally limited triaxial work to about $P=1 \mathrm{GPa}$, with most triaxial machines, in fact, having maximum design pressures of 0.3-0.5 GPa (Paterson 1978). At higher pressures, the solid pressure medium piston-cylinder apparatus is used. The solid-medium press used most widely for geological applications is the Griggs apparatus (Griggs 1967), adapted from the hydrostatic solid-medium pistoncylinder apparatus (Boyd and England 1960), and subsequently improved to provide a stress resolution of a few $\mathrm{MPa}$ (Borch and Green 1989; Green and Borch 1989; Tingle et al. 1993). Since a solid medium has a finite shear strength, measurements are limited to materials

Figure 1. The Griggs triaxial deformation apparatus, including the yoked pistons to hold pressure constant during deformation. As piston $\mathrm{P}_{1}$ moves against the sample, piston $\mathrm{P}_{2}$ automatically withdraws from the vessel at the same rate, holding the pressurized volume constant. After Griggs (1936), Fig. 2; reproduced with permission. 


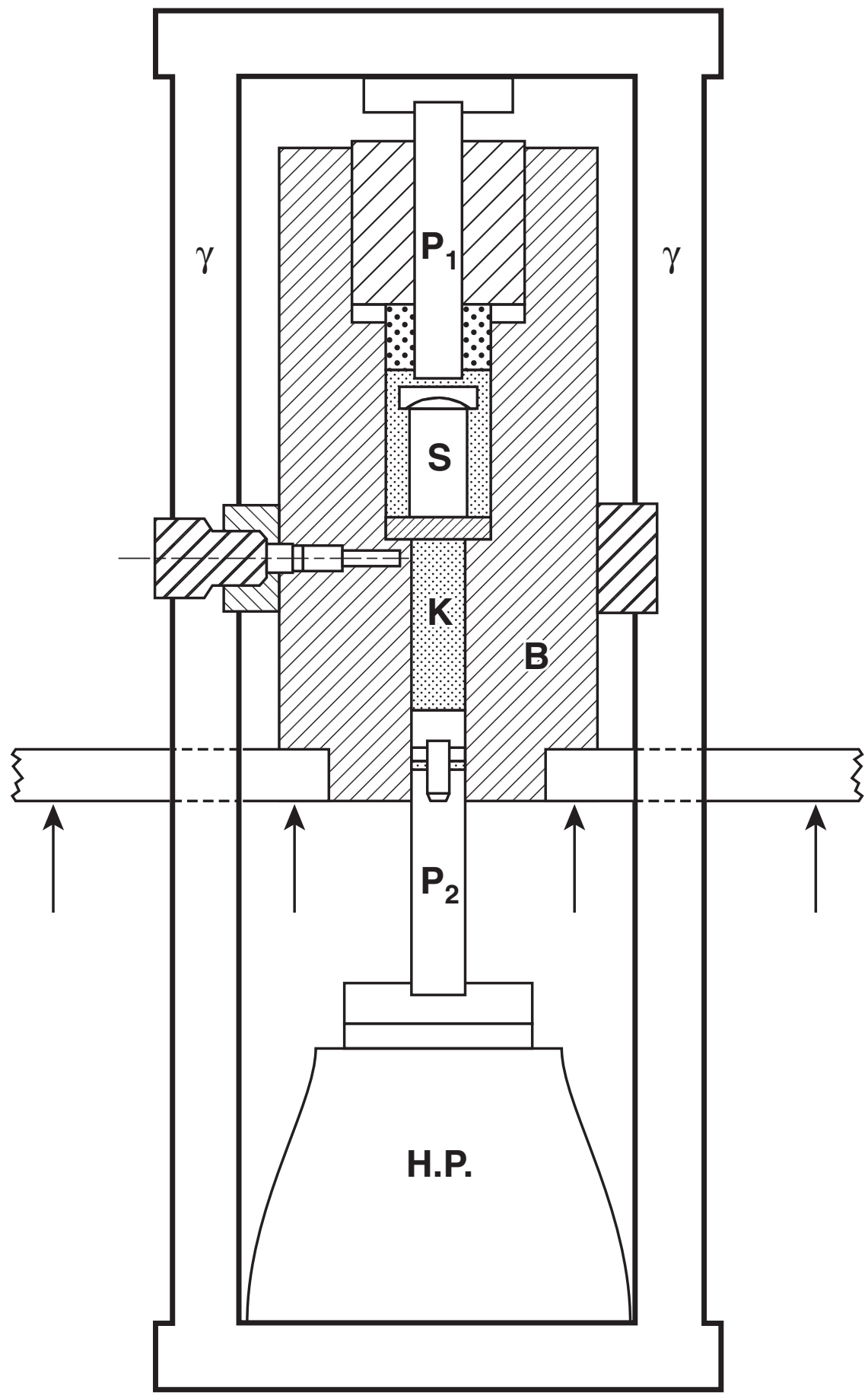

Figure 1 
that are stronger than the medium, so very low strain rates and temperatures near the sample melting point must be avoided, but pressures of several GPa are now easily accessible. The Griggs apparatus has been regularly used to $1.5-1.8 \mathrm{GPa}$, and to a maximum pressure of $4 \mathrm{GPa}$.

A recent development is an apparatus built by Getting (Getting 1998; Getting and Spetzler 1993) that combines aspects of the solid-medium and fluid-triaxial deformation machines. Getting's is a piston-cylinder device that features precision packings that are sufficiently tight to contain solid argon (an exceedingly weak solid) but sufficiently reproducible in their frictional behavior that much of the uncertainty in stress introduced by piston friction can be accounted for by calibration. Getting (1998) claims pressure uncertainty at $P=3 \mathrm{GPa}$ at the extraordinary level of a few MPa. If this resolution applies also to the sum of $\sigma_{1}+P$, as expected, it may mean that rock strengths can be measured with a resolution that is comparable to that of many triaxial gas rigs that operate at far lower pressures.

The practical pressure limit for all cylindrical machines is around $5 \mathrm{GPa}$. While the pressure capacity of a hollow cylinder (or any shape, for that matter) is a function of wall thickness and therefore virtually unlimited (Eremets 1996), the same is not true for the piston. As one approaches $P=5 \mathrm{GPa}$, the stress required to drive the piston into the pressure cylinder (which must be $5 \mathrm{GPa}$ plus the stress to overcome packing friction plus the strength of the sample) causes pistons constructed of even the strongest of steels to creep. Pistons will swell and seize in a short amount of time. Note that this 5-GPa limit exists for hydrostatic piston-cylinder vessels as well. Bridgman reached higher pressures in hydrostatic vessels by confining the entire piston and cylinder in a larger vessel, thus raising $\sigma_{3}$ on the piston to the pressure in the outer vessel and allowing the piston to apply correspondingly more stress to the inner vessel without exceeding its strength limit. His vessel-inside-vessel multi-staged devices have been used to reach pressures of at least $10 \mathrm{GPa}$ (e.g., Bridgman $(1942 ; 1948)$ ). Because of the complexity of those vessels, however, they are not well suited to being instrumented for deformation studies.

\section{GPa and above: Anvil devices}

A much simpler method for achieving an elevated $\sigma_{3}$ is to give the pistons a tapered shape. (The change from rectangular to trapezoidal section also merits a name change: from piston to anvil.) Now a component of the force applied in the $\sigma_{1}$ direction at the larger end of the piston/anvil is directed in the $\sigma_{3}$ direction at the smaller end (platen face), making $\sigma_{3}>0$ at the platen face. The drawback of this solution is, of course, that one cannot expect to contain the pressure medium over large displacements in a conventional cylindrical pressure vessel if the sides of the (moving) anvil are tapered.

Sealing against a moving tapered surface can be achieved for small displacements through use of a crushable gasket material; so if one is satisfied with anvil displacements that are less than gasket thickness, one can reach high values of $\sigma_{1}$ at the platen faces without exceeding the failure criterion of the anvils. The first application of this principle is generally credited to Bridgman's (Bridgman 1952a) opposed-anvil device, which is capable of $10 \mathrm{GPa}$, although Bridgman used the same principle many years earlier in confining samples in a 5-GPa shear apparatus (Bridgman 1935a). It is also the underlying principle of the Drickamer cell (see below). Note that the original purpose of anvil displacement in these designs was pressure generation rather than sample deformation, although as we will see below, the possibility of the latter was not ignored.

A slight variation, which allows for larger volumes at a given pressure, is to combine the function of pressure container and pressure generator by clustering several anvils in a quasi-spherically symmetric pattern. These are called multianvil devices. Four-anvil 
tetrahedral (Hall 1958; Houck and Hutton 1963; Lloyd et al. 1959), six-anvil cubic (Carter et al. 1964; Houck and Hutton 1963; Osugi et al. 1964), and eight-anvil octahedral (Kawai and Endo 1970; Walker et al. 1990) have been constructed. The eightanvil devices are multistage, with the eight anvils of the final stage contained within the working volume of an intermediate cubic stage. They are often referred to, therefore, as $6 / 8$ devices. Hydraulic actuation of the several anvils was sometimes independent or in opposed pairs (Carter et al. 1964; Hall 1958), but better symmetry of anvil displacement was achieved, along with higher pressures, when the anvils were driven by wedge-shaped guide blocks that then required only one hydraulic actuator (Lloyd et al. 1959). There are at least two versions of the wedge-type cubic multianvil device: a split-sphere device (Houck and Hutton 1963) and the DIA (Osugi et al. 1964; Shimomura et al. 1985); the latter will be describe below in detail.

Wedged guide block machines achieve high pressures more reliably than machines with multiple actuators, and for that reason they have proliferated, especially the DIA and 6/8 devices (e.g., see review by Onodera (1987)). Note, however, that their design function is exclusively hydrostatic pressure generation. Advances in high-pressure rheology required that the quasi-isotropy within the pressurized volume be broken; those advances are the main subjects of this review.

Another reason for the recent proliferation of multianvil devices is a serendipitous advantage they have over pressure cylinders: optical and/or X-ray transparency along a line of sight leading directly to and from the sample (Fig. 2). In the case of diamond (or sapphire) anvil cells, the anvils themselves are transparent. For devices where anvils are opaque, lines of sight are always available in the narrow gaps between anvils, and the gaskets compressed in those gaps can be made of X-ray-transparent weak material (e.g., pyrophyllite). The analytical potential of high-energy X-rays and their recent availability at very bright synchrotron sources has been the motivation for development of a new generation of deformation machines that have the pressure capacity of anvil devices and the lowered symmetry of deformation machines.

\section{MEASUREMENT METHODS AT HIGH PRESSURE}

\section{Stress measurement}

Measurement of deviatoric stress is key to quantifying the rheological properties of a material. Stress provides information about strength relative to the laboratory time scale. Stress, measured in conjunction with ductile strain, can yield flow laws within the context of the state of the sample. The crucial ingredient to facilitate such studies is the development of a stress meter, or piezometer.

In experiments operating below $5 \mathrm{GPa}$, the deforming force is applied directly to the sample and various strategies are designed to measure the magnitude of this deforming force. Stress is then given as the ratio of force to sample area. Typically, a load cell placed in series with the deforming piston defines this force along the deforming column. Combinations of friction between the force gauge and the sample and forces that support the piston, such as in the multianvil system, continually degrade the ability to relate a measured force to sample stress. These problems begin to interfere with the resulting accuracy at $0.3 \mathrm{GPa}$ and completely overwhelm the measurements by $5 \mathrm{GPa}$.

A revolutionary technique for stress and strain measurements under high-pressure (and temperature) conditions in situ has now become feasible using high-energy X-rays generated by a synchrotron radiation facility. These new tools are just now being explored and their limitations defined. The exceptional quality of these tools rests in the fact that they are directly monitoring the sample. Stresses are measured in the sample. 
Figure 2. Sample assembly in the DIA cubic apparatus, shown with three of the six anvils removed and with the sample assembly itself, i.e., the cube, shown in cross section. Analytical synchrotron $\mathrm{X}$-rays enter and exit along a horizontal line through the sample and normal to the section as shown here. The X-rays pass through the vertical gaps between anvils. Drawing by M. Vaughan.

Different positions in the sample can be isolated to test for stress uniformity. Measurements of stress and strain can be time resolved with a precision of about one minute. Strain is also obtained by images of the sample. Distribution of strain with position and time can be defined. All of this is done with a current accuracy of a few tens of $\mathrm{MPa}$ in differential stress and $10^{-4}$ in strain.

Strategies for measuring stress are found through use of the X-ray diffraction or pressure-sensitive probes (such as ruby fluorescence), and are being made available by exploiting powerful synchrotron X-ray sources. The basic principle of X-ray piezometers is that lattice strain is sensitive to elastic strains and not to plastic strains. Changes in distances between atoms are expected to balance stress fields that are present. These stress fields may be created by dislocations or external forces, but departures from the lattice spacing that the material experiences at zero pressure and stress are sensibly related to the stress field within the sample. Furthermore, the coupling between the strain and stress is expressed by the elastic moduli. There are two styles of X-ray piezometers, one based on stress gradient and the other based on strain anisotropy. 


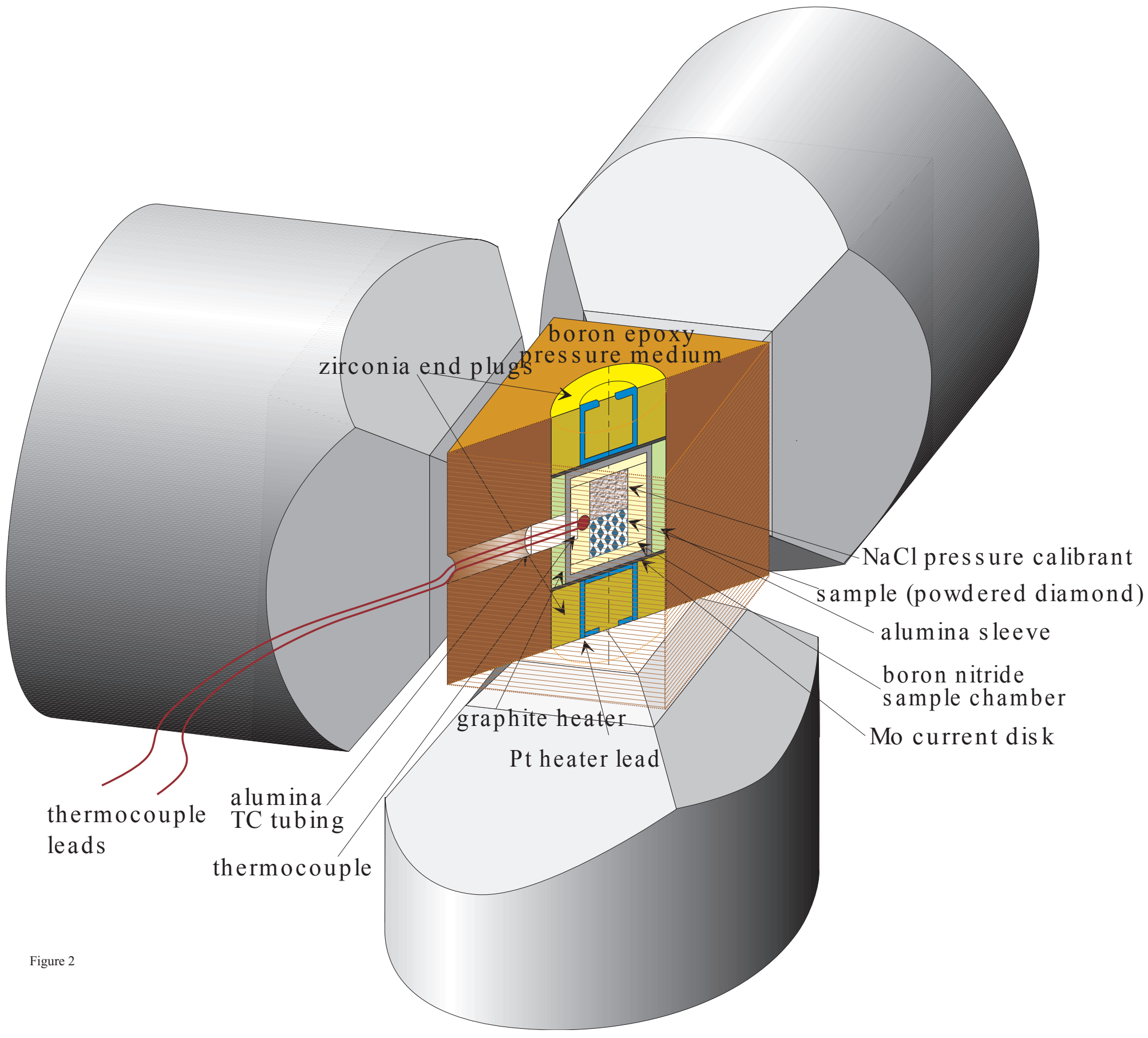


Stress gradient. Where the inertia term can be neglected, variation in the stress field is restricted to obey the mechanical equilibrium relations:

$$
\sum_{j=1}^{3} \frac{\partial \sigma_{i j}}{\partial x_{j}}=0
$$

Three equations, one for each value of $i$, represent the vector force balance equations. The first of these equations is:

$$
\frac{\partial \sigma_{11}}{\partial x_{1}}+\frac{\partial \sigma_{12}}{\partial x_{2}}+\frac{\partial \sigma_{13}}{\partial x_{3}}=0
$$

Thus, if there is a variation of the normal stress, $\sigma_{11}$, with $\mathrm{x}_{1}$, there must also be a variation of the shear stresses, $\sigma_{12}$, in the $\mathrm{x}_{2}$ direction and/or a variation of $\sigma_{13}$ in the $\mathrm{x}_{3}$ direction. The peak magnitude of the shear stress is related to the variation of the normal stress (or pressure) and the length scales of two types of stress. This has been exploited in the diamond anvil cell geometry by measuring the radial gradient of pressure to deduce the axial (along the axis of the diamonds) variation of shear stress. This is illustrated in Figure 3. The shear stress, $\tau$, vanishes along the center plane of the sample and along the central axis of the cell where $\partial \mathrm{P} / \partial \mathrm{r}$ is zero. The maximum shear stress is at the samplediamond interface and is given by

$$
\tau_{\max }=(\partial \mathrm{P} / \partial \mathrm{r})_{\max } \mathrm{d} / 2
$$

where $\mathrm{d}$ is the thickness of the sample. This technique was developed by Sung et al. (1977) and was further used by Meade and Jeanloz (1988a,b; 1990). These experiments relied on ruby dust distributed across the sample as a pressure marker for delineating the

Figure 3. Illustration of stress field in a diamond-anvil cell. The radial distribution of pressure, illustrated at the top, is coupled to the shear stress distribution along the axial direction. Force couples indicate the sense of the shear stress distribution within the cell. 
Pressure

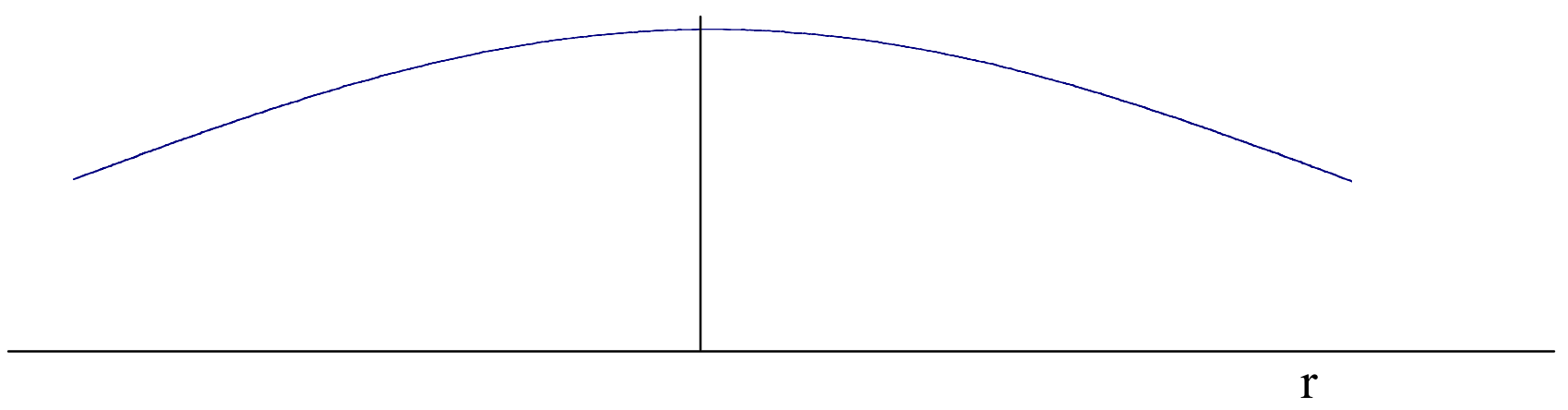

Diamond Loading

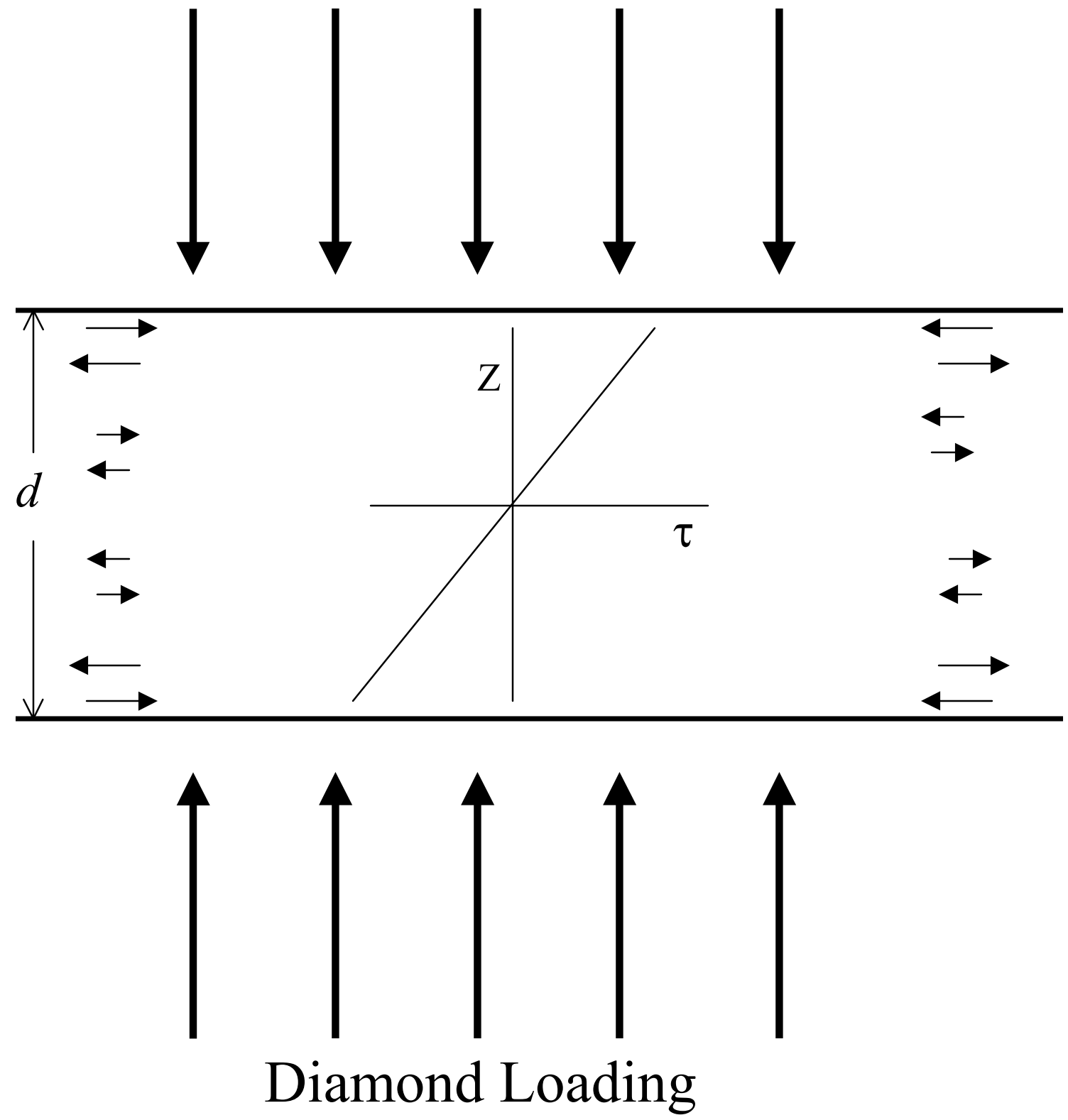

Figure 3 
pressure gradient. As Wu and Bassett (1993) illustrate, the pressure marker can also be an X-ray diffraction standard such as gold. The objective is to measure the radial pressure profile to define the shear-stress distribution. Often, the peak shear stress is taken as the yield point of the solid.

Stress gradients coupled with deviatoric stress affect the diffraction peak shape as described in detail by Weidner(1998) and Weidner et al. (1998). In this case, heterogeneities in the stress field broaden the diffraction peaks. Since the length scale of stress variation is the same for normal and shear stress, then the magnitude of the heterogeneity for all stresses is similar. Weidner et al. (1998) model the relationship between the observed strain (that creates the broadened diffraction peak) and the stress magnitude and conclude that Young's modulus is the appropriate coupling elastic modulus, or:

$$
\sigma_{\text {differential }}=\mathrm{E} \varepsilon_{\text {differential }}
$$

where the differential strain is deduced as the "strain broadening" contribution to the peak profile. Since for the peak-broadening measurement, the full-width, half-height of the diffraction peak is used to define the strain broadening and represents roughly twothirds of the scattering diffraction planes, then most of the sample must be experiencing the inferred shear stress. Deviatoric stress can originate from dislocations, from heterogeneities in material or properties, or any number of sources. Peak-broadening analysis is often used in commercial applications to determine stresses induced by processing. For example, internal stress generated by welding is often analyzed by this method. In high-pressure studies, the stress is most commonly generated by compressing powdered samples (e.g., Chen et al. 1998). As a rheological tool, this approach resembles an indentation experiment where the indenter is the same material as the sample. Weidner et al. (1994) studied the high-temperature and -pressure strength of diamond with this method. Chen et al. (1998) studied plastic deformation of olivine, wadsleyite, and ringwoodite. Flow can also be studied with this piezometer, with the constraint that this becomes a relaxation experiment (Weidner et al. 2001).

Strain anisotropy. A uniaxial stress will introduce elastic strains in the sample. The strain parallel to the axis of compression will generally be larger than the strain perpendicular. The material's elastic moduli quantitatively define the relationship between these strains and the imposed stresses. X-rays sample the distance between lattice planes (called $d$ spacing) whose normals are parallel to the diffraction vector, which is the bisector between the incident X-ray and the detector. The lattice spacing is insensitive to strain history (plastic strain) but reflects the elastic strain field. Figure 4 illustrates a Debye ring that would be observed from a powder sample in a stress field using a monochromatic Xray beam and an area detector. The lattice spacing is related to the distance from the center of the image to the Debye ring through Bragg's law. For the angle $\Psi$ of zero, the lattice spacing reflects that measured parallel to the $\mathrm{x}_{1}$ direction. For $\Psi$ of ninety degrees, the X-rays are sampling grains aligned parallel to the $\mathrm{x}_{2}$ direction. The strains deduced from these measurements compared with ambient conditions are related to the stresses by:

$$
\varepsilon_{i j}=\sum_{k=1}^{3} \sum_{l=1}^{3} s_{i j k l} \sigma_{k l}
$$

where $\mathbf{s}$ is the elastic compliance tensor.

In the actual case, more than one set of diffraction planes will produce Debye rings similar to the one illustrated here. Each will be approximately circular in shape, but distorted by the stress field. The amount of distortion may be different if the elastic 
Figure 4. X-ray diffraction line on a two-dimensional detector from a sample under stress. The distance from the center to the ring defines the scattering angle, $2 \theta$, and hence the lattice spacing through Bragg's law. The presence of the stress field distorts the Debye ring from its normally circular shape. The usual stress field is axisymmetric, with $\sigma_{2}=\sigma_{3}$. The diffraction vector at $\psi=0$ lies at an angle $\theta$ from the direction of $\sigma_{1} . \theta$ is typically only 3 to 4 degrees.

compliance tensor is not isotropic. To evaluate this in detail, we need to be careful to account for the crystal orientation when defining the compliance tensor. Thus, in a nonhydrostatic stress field, crystallographic planes with different orientations change their spacing by different amounts. For cubic crystals, the relationship between the $\Psi$ dependence of strain and the differential stress, $\Delta \sigma$, is given by:

$$
\varepsilon_{\mathrm{hkl}}(\Psi)=\Delta \sigma / 3\left\{1-3 \cos ^{2} \Psi\right\}\left\{\left(\mathrm{S}_{11}-\mathrm{S}_{12}\right)[1-\Gamma(\mathrm{hkl})]+\mathrm{S}_{44} \Gamma(\mathrm{hkl}) / 2\right\}
$$

Each diffraction peak corresponds to a set of lattice planes defined by the Miller index, hkl. In an anisotropic crystal, the elastic moduli will depend on the particular set of planes that are considered. The function, $\Gamma(\mathrm{hkl})$, varies from 0 to 1 and accounts for this variation as it multiplies the different elastic compliance moduli, $S_{\mathrm{ij}}$. Thus, for a particular diffraction plane, hkl, the right hand bracket contains a constant value. Then $\varepsilon$ varies in a fixed manner with the angle, $\Psi$.

The above expression is based on the assumption that each grain experiences the same stress field, or the Reuss condition. A similar relation can be derived by assuming that each grain satisfies the Voigt condition of iso-strain. In this case the equation is altered by replacing the elastic shear compliances, $S_{44}$ and $\left(S_{11}-S_{12}\right)$ by their Voigt equivalent. This relation for cubic symmetry is generalized by Singh et al. (1998) for all crystal symmetries.

This phenomenon gives rise to a couple of strategies for a piezometer. Stress has a manifestation even for a single value of $\Psi$. For an elastically anisotropic material, the strain for a given crystallographic plane will depend on the orientation of crystal with respect to the applied stress and the stress magnitude. Therefore by measuring the strain for several diffraction peaks corresponding to different orientations, one can determine the stress magnitude using the known elastic constants of the crystal. Such a measurement can now be done in a multianvil high-pressure apparatus using a synchrotron X-ray source (Weidner et al. 1992). The accuracy relies on knowing the elastic anisotropy at the pressure/temperature conditions of the experiment.

A more robust strategy comes from mapping out the $d$ spacing as a function of $\Psi$. The values of the elastic moduli are still required, but the details of anisotropy are less critical to yielding precise measurements. With a precision of $10^{-4}$ for the lattice spacing 


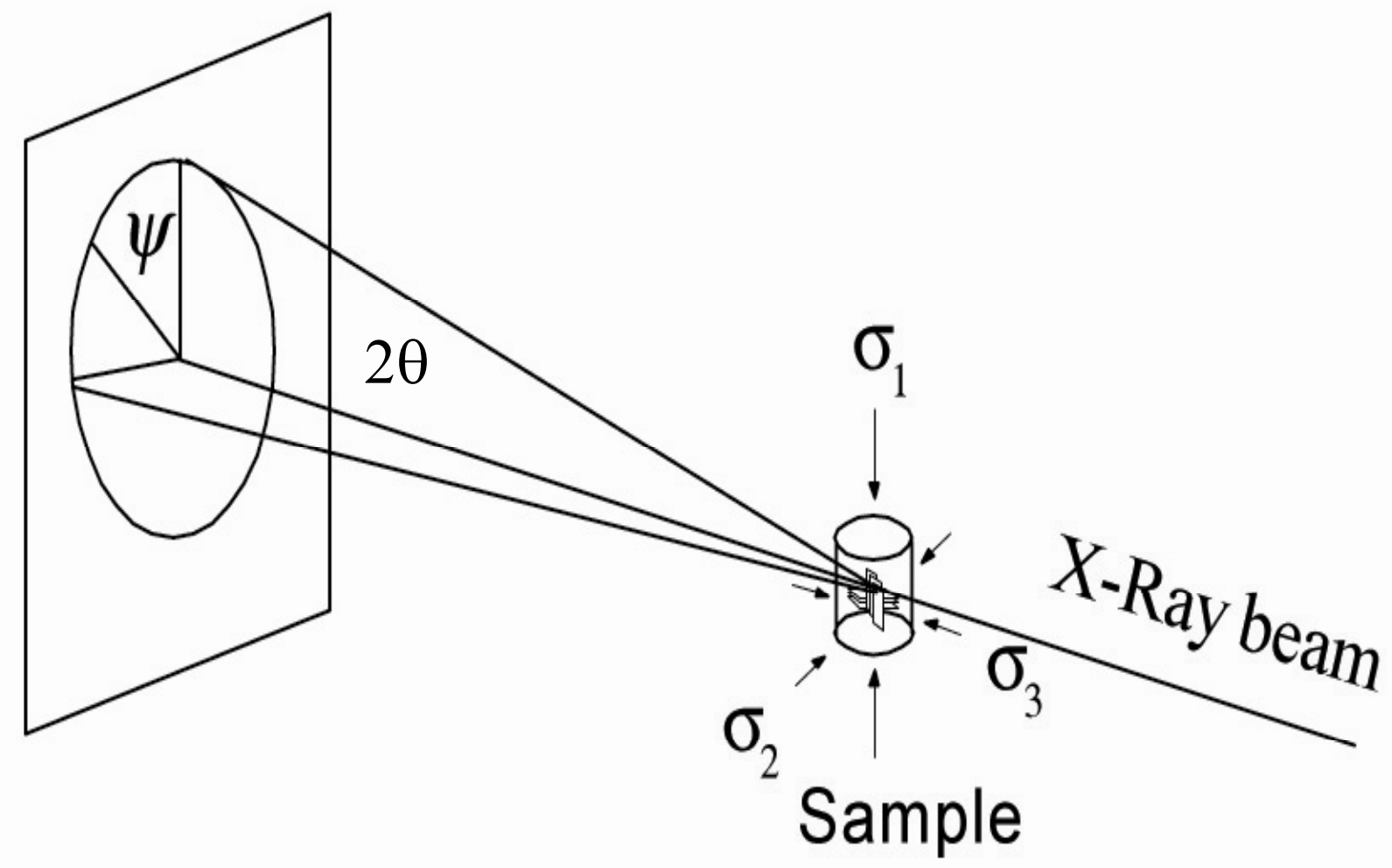

Figure 4 
and a typical elastic modulus of $200 \mathrm{GPa}$, it should be possible to resolve differential stresses of $\sim 20 \mathrm{MPa}$. Singh has reduced the elasticity equations to a closed-form solution for several crystal symmetries and has given a concise summary (Singh et al. 1998).

This methodology was first applied in a diamond-anvil cell by Kinsland and Bassett (1977) by passing the X-ray beam through a Be gasket, perpendicular to the axis of the diamonds. The multianvil system has not been accessible by this technique because the anvils themselves cast a shadow and limit the range of $\Psi$ where the diffracted signal can be observed. However, we have recently experimented with cubic BN anvils. These are nearly as hard as diamond but are about an order of magnitude less expensive. Furthermore, they are transparent to X-rays. Thus, by using these anvils it is now possible to observe diffraction spectra in any plane relative to the incident beam.

Here we have illustrated this tool with monochromatic X-rays and angle dispersive measurements. Energy dispersive methods are equally valid for these measurements and are sometimes advantageous. Spectra need to be collected for different values of $\Psi$ through collimators that fix the two-theta value. Weidner's group has designed and used a conical slit system for this purpose as illustrated in Figure 5. Two concentric cones, whose angle is the desired diffraction angle for white, energy dispersive diffraction, create the slit itself. Solid-state detectors with energy discrimination are placed at specific values of $\Psi$ behind the slit system. Each detector is calibrated independently. Lattice spacings are used as illustrated above to define the differential stress.

Figure 5. Conical slit assembly in use at National Synchrotron Light Source. The cone defines the two-theta angle and is constructed by two concentric cones with this angle. The X-rays that pass between the two cones originate from the sample at that angle as all other X-rays are blocked by the cones. Multiple energy discriminating detectors, located behind the slit assembly, record the X-ray signals. A YAG crystal located inside the slit assembly fluoresces in the X-ray beam, emitting visible light where an X-ray photon passes. The visible light is magnified by a microscope assembly and recorded by a digital camera.

\section{Strain rate measurement}

Synchrotron X-rays can also be used to measure sample length. Direct images of the sample can be obtained using an incident beam whose dimensions are larger than the sample. Platinum or gold foils above and below the sample are easily viewed. The X-ray image is obtained by projecting the X-ray onto a fluorescent screen that is viewed with a CCD camera though a magnifying system. A typical image is shown in Figure 6. The 


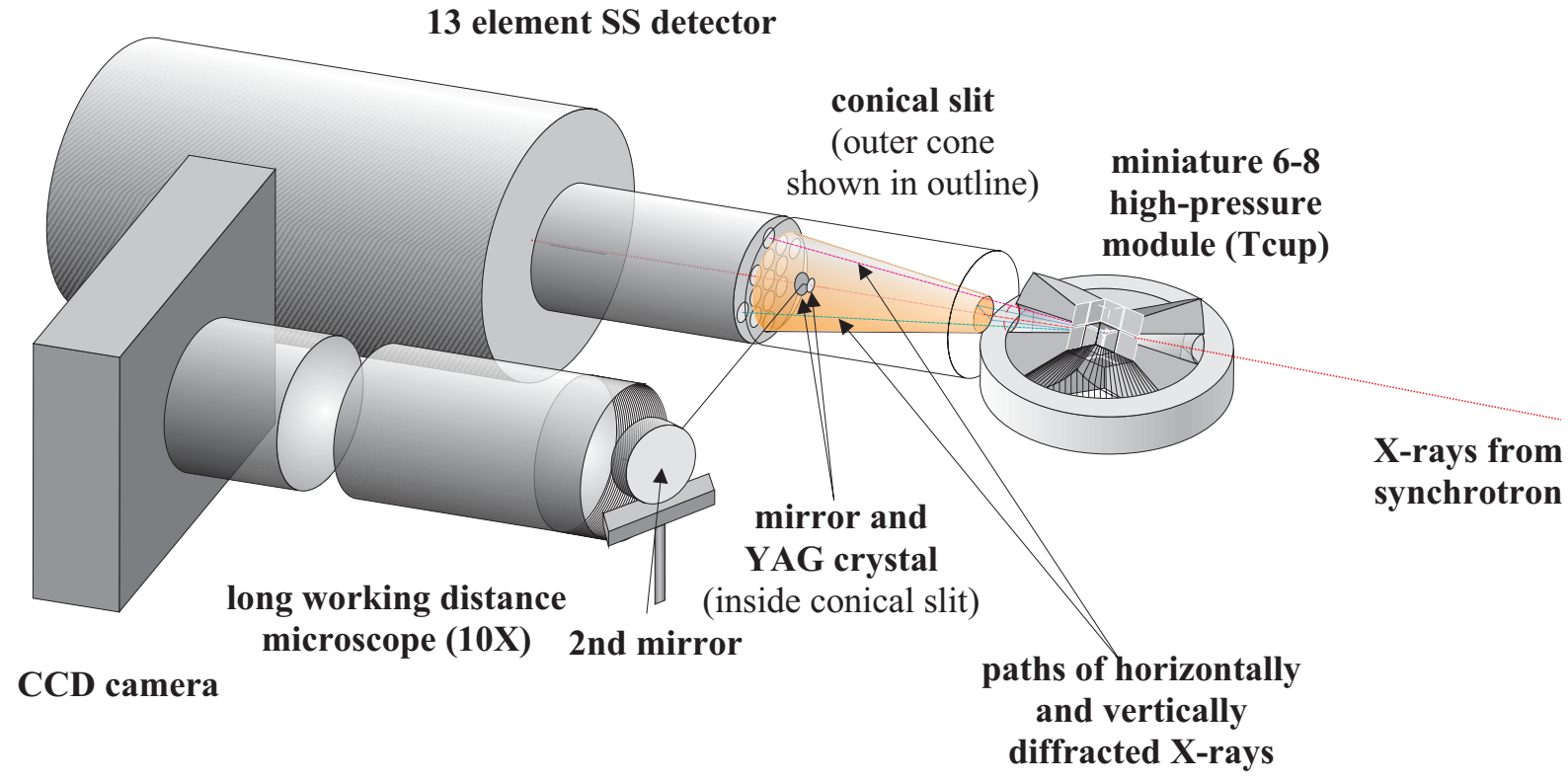

Figure 5 
horizontal lines in this image are gold discs placed at the ends of the sample. The black edges are the shadows of the opaque tungsten carbide anvils. Cubic boron nitride anvils are transparent and can be used to view the entire sample because they do not cast such a shadow. Figure 5 illustrates the position of the microscope for these measurements. The fluorescent screen is located on-axis inside the conical slit system. We determine that, by comparing two images, strains of $10^{-4}$ can be measured. For images taken 100 seconds apart, this allows resolution of strain rates of $10^{-6} \mathrm{~s}^{-1}$.

Figure 6. X-ray shadowgraph of $\mathrm{MgO}$ sample during compression. The horizontal dark lines are images of gold foil that bound the sample whose length is $\sim 1 \mathrm{~mm}$. The horizontal dimension is defined by the opening between the high-pressure anvils. The shadow across the top is caused by a platinum foil wrapped around the end-plug.

The X-ray image yields the total strain of the sample, which is the sum of the elastic and plastic strain or:

$$
\varepsilon_{\mathrm{ob}}=\varepsilon_{\mathrm{el}}+\varepsilon_{\mathrm{pl}}
$$

In an experiment both the elastic and plastic components may vary with time. Thus, to deduce the plastic strain rate, it is necessary to account for the variations of all strain components. The elastic strain, however, is reflected in the diffraction pattern and can be represented by a unit cell length, a. This cell-edge length is obtained from analysis of all of the diffraction peaks recorded with a diffraction vector parallel to the sample axis. In elastically isotropic samples, there is no ambiguity in defining this value. Anisotropic samples in a differential stress field will exhibit lattice spacings that reflect the aggregate properties. In this case, an average cell dimension based on several diffraction peaks should be obtained. We define the Kung ratio, $R_{k}$, as:

$$
R_{k}=\left(d / d_{0}\right) /\left(a / a_{0}\right)
$$

where $d$ is the length of the sample measured with an image and $a$ is a unit cell length measured from diffraction; the zero subscript refers to a reference value such as ambient conditions. This ratio, which is normalized to one, represents the length of the sample in the units of the cell dimension. This number is proportional to the number of unit cells that make up the length. The Kung ratio remains constant if all deformations are elastic. Thermal expansion, pressure, and stress will not change the Kung ratio if the sample responds elastically. Plastic strain changes the Kung ratio. In this case the number of unit cells that define the length changes. Thus, the Kung ratio can be used as the proxy for plastic strain, or:

$$
\varepsilon_{\mathrm{pl}}=\left[R_{\mathrm{k}}(2)-R_{\mathrm{k}}(1)\right] / R_{\mathrm{k}}(1)
$$

where (1) and (2) represent two states of the material.

Strain is not always uniform in the sample. Visual tools as described here provide opportunity to map the strain field as a function of space and time during the experiment. Vaughan et al. (2000) demonstrate that one can embed strain markers in a sample during preparation and view the position of these markers during the experiment. Figure 7, from this work, shows a grid made from a TEM gold sample grid, in the sample at different $\mathrm{P}$, $\mathrm{T}$ conditions. The sample was a sintered olivine cylinder. The cylinder was sliced into 


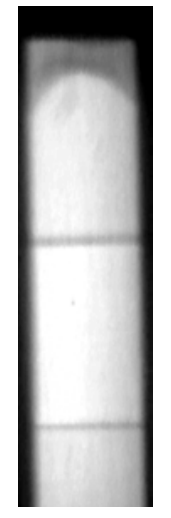

Figure 6 
two halves, the grid inserted, and the sample halves placed back together. The sample assembly included hard end-plugs in a DIA apparatus, so as to produce shortening during compression. The grid size is initially about 70 microns. With these images, it is possible to map the strain as a function of position in the sample and as a function of time by comparing successive images.

Figure 7. A TEM gold grid embedded on the central plane of a cylindrical sample. Images at different conditions illustrate different amounts of strain. The initial pressurization distorted the grid because surfaces of sample assembly parts were not perfectly flat. Subsequent strain can be mapped by following specific strain markers.

\section{MODERN TECHNIQUES FOR DEFORMATION AT HIGH PRESSURES}

In current technology, deformation at $P>10 \mathrm{GPa}$ is the realm of anvil devices. We describe in detail four methods currently in use or in active development: (1) sample assembly modification to hydrostatic multianvil devices, (2) sample assembly modification to the DAC, (3) the D-DIA, and (4) the RDA.

\section{Sample assemblies}

Sample assemblies used in these machines share several common features of those used in hydrostatic devices, serving common requirements such as high pressure, high temperature, and control of chemical activity in the sample. With the exception of the specialized environment of the DAC, these common features include a pressure medium, such as soft fired pyrophyllite, $\mathrm{ZrO}_{2}, \mathrm{MgO} / 5 \% \mathrm{Cr}_{2} \mathrm{O}_{3}$, castable ceramic or epoxy; a furnace, for example, metal foil, $\mathrm{LaCrO}_{3}$, or graphite; and a soft medium surrounding the sample itself, for example, $\mathrm{BN}, \mathrm{MgO}, \mathrm{NaCl}$, or glass (the latter two occasionally as liquids). Descriptions of such assemblies can be found in, e.g., Kawai and Endo (1970), Liebermann and Wang (1992), Walker(1991), and Weidner et al. (1992). For control of chemical environment the sample can be encapsulated along with buffering compounds (see e.g., Rubie et al. 1993).

Non-hydrostaticity is introduced by introducing hard platens that impart shear or 


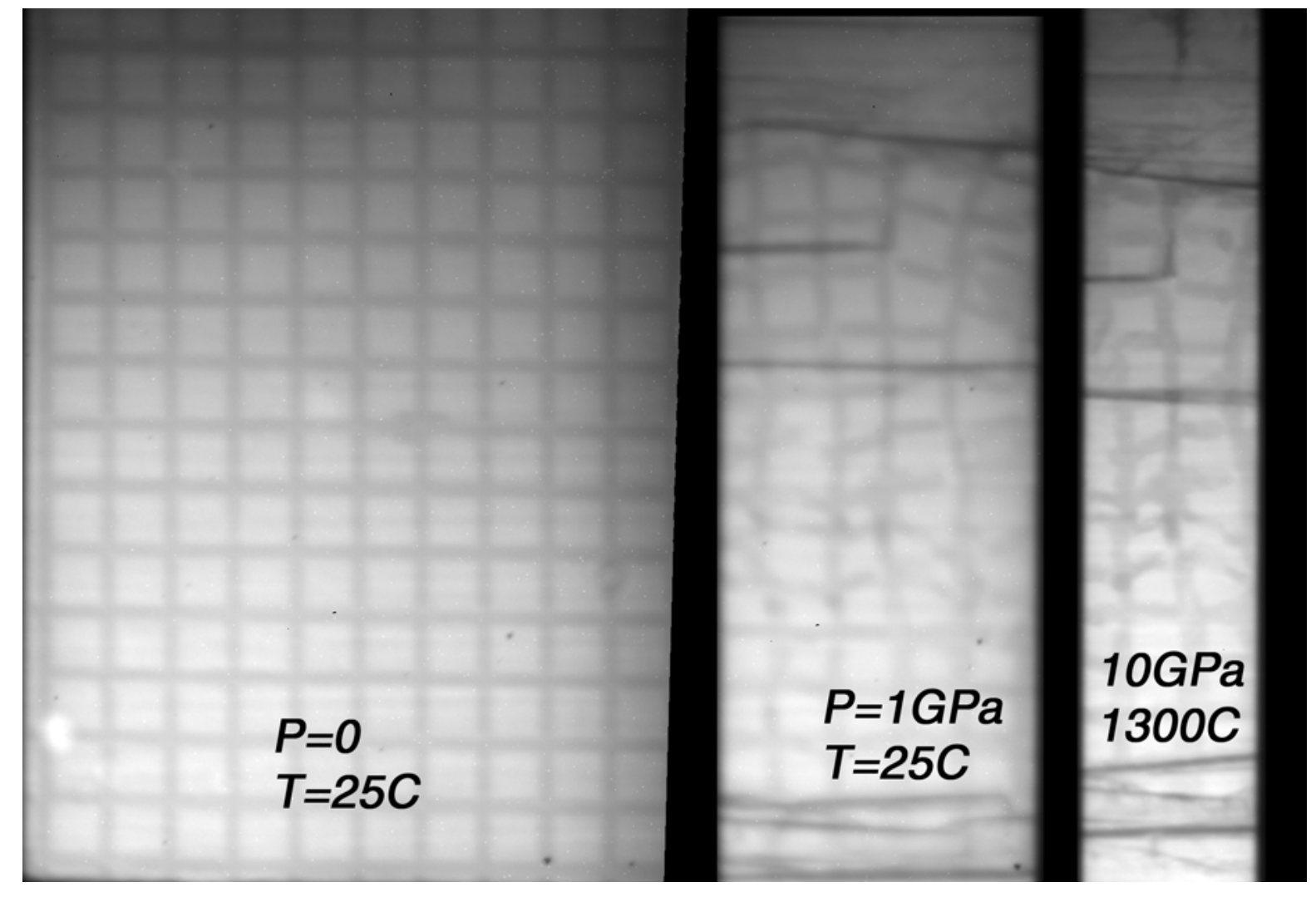

Figure 7 
normal stresses to the sample. Several of the methods for achieving this are compared in Figure 8. They provide axisymmetric deformation in the multianvil devices (Figs. 8a-8c), simple shear in the multianvil (Fig. 8d), axisymmetric deformation in the DAC (Fig. 8e), and rotational (approximately simple shear) deformation in the RDA (Fig. 8f). We compare these assemblies briefly here and in more detail below.

Figure 8. Starting $(P=0)$ configurations of sample columns used for deformation experiments at very high pressures. For simplicity, details such as sample encapsulation, thermocouples, and furnace electrodes are not shown. (a) Stress relaxation. The sample is loaded elastically during pressurization. The furnace and outer and inner pressure media shown here also apply to (b)-(d). (b) Constant stress/strain rate tests. The porosity within the crushable ends of the column takes up most of the column shortening during pressurization. (c) Specialized version of (b), with a fluid in the column assuring strictly hydrostatic loading of the sample during pressurization. (d) Simple shear, high strain. (e) Column for deformation in the diamond-anvil cell. Use of a separate piston is optional, i.e., the diamond anvils can also act as pistons. In some applications, a ruby piston can also serve as a differential stress gage. (f) Assembly for the rotational Drickamer apparatus, also shown in Figure 13.

The adaptations to the DIA and 6/8 discussed in the next section use a column such as shown in Figure 8a, in which the source of deviatoric stress in the sample is the closure of the anvils during initial pressurization. At the scale of Figure $8 \mathrm{a}$, the shortening of the column (elastic plus inelastic) is approximately $20 \%$ of the column length (Durham and Rubie 1998), so the "crushable" material at the ends of the column, present to protect thermocouple wires and furnace electrodes, has a porosity somewhat less than $20 \%$ of the column length. To avoid deforming the sample during pressurization in devices capable of deforming at fixed pressure (currently only the D-DIA) one uses a column such as in Figure $8 \mathrm{~b}$, where the length of crushable material is sufficient to absorb all anvil displacement during pressurization When a strictly hydrostatic environment is required during pressurization, such as when the sample is a fragile single crystal, it is also possible to create a liquid cell around the sample before the pressurizing load is applied (Fig. 8c) (Durham, unpublished results). 


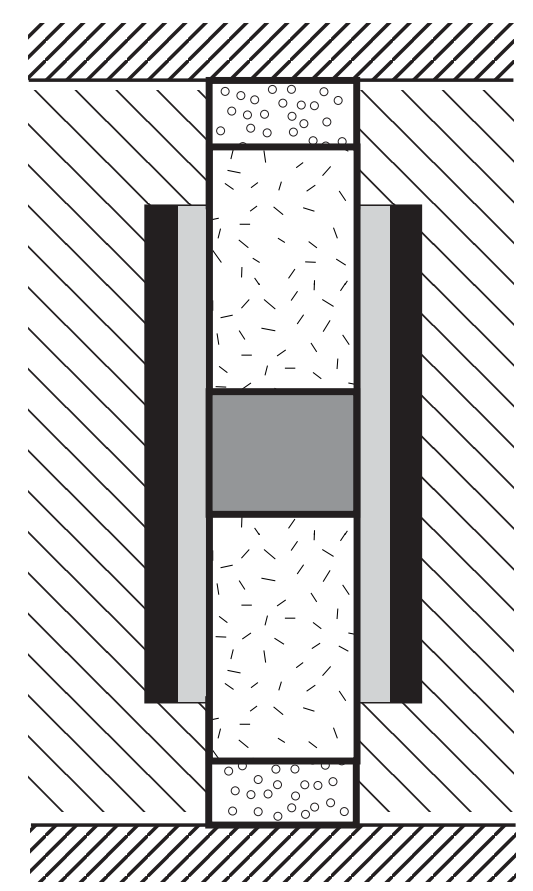

(a)

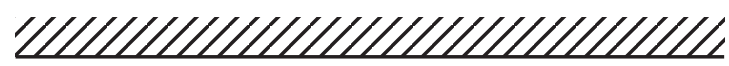

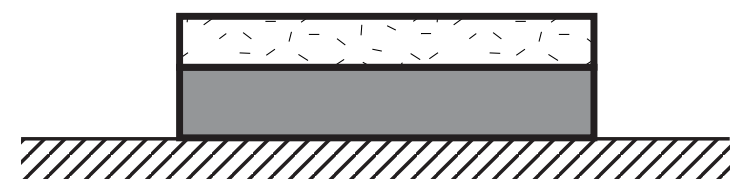

(e)

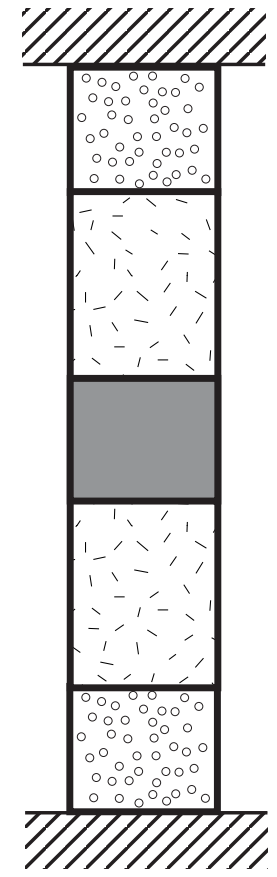

(b)

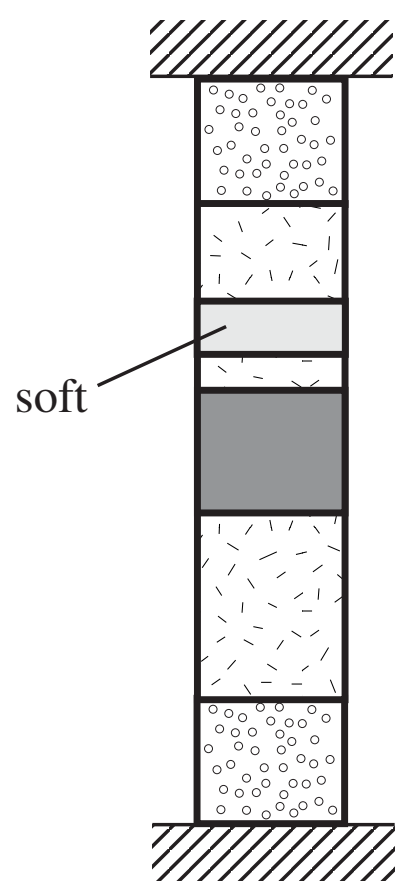

(c)

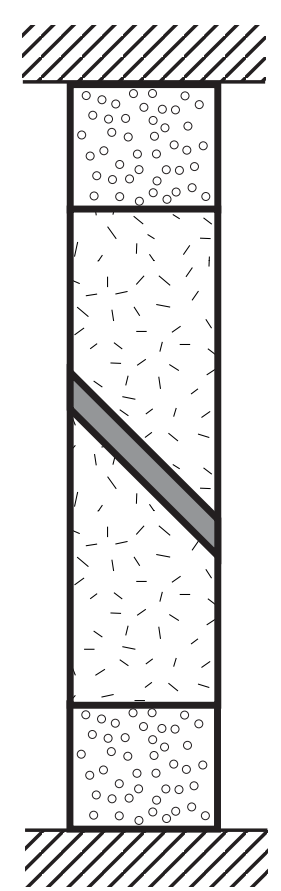

(d)

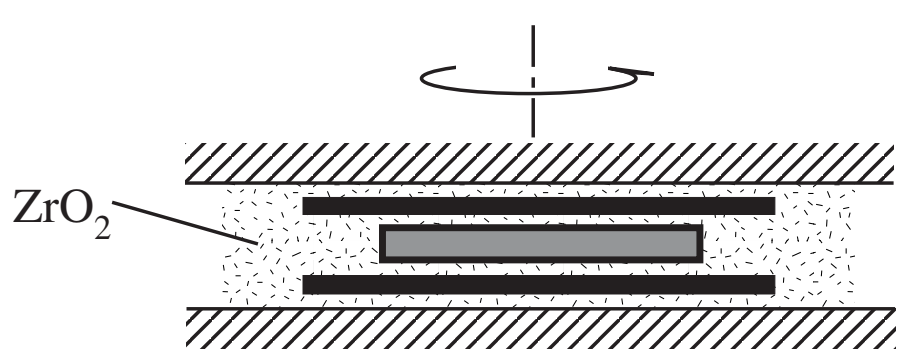

(f)

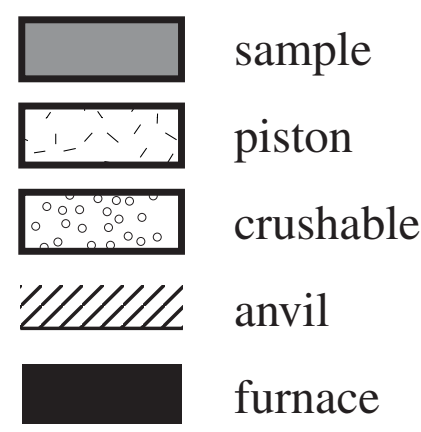

soft $\mathrm{P}$ medium

outer P medium 
Figure $8 \mathrm{~d}$ shows the method for converting axial displacement to high-strain, simpleshear deformation in the multianvil cell (Karato and Rubie 1997). As discussed below, this design was first introduced as a method for imposing larger strains in the stressrelaxation mode of the hydrostatic multianvil device. However, it can also be used in the D-DIA, presumably with slightly more crushable material at the ends, to carry out constant stress or constant strain-rate tests to very high simple-shear strains.

The very simple deformation assembly for the DAC (Fig. 8e) reflects the greatly reduced available volume and the fact that laser heating can be used in place of a resistance heater to achieve local heating of the sample. Finally, the assembly for the RDA (Fig. 8f) is the rotational analog to the axisymmetric columns in Figures 8a-8c. Because rotational deformation and pressurization are entirely decoupled in the RDA, there is no need for crushable material in the cell.

\section{Modifications to the sample assembly in a multianvil press}

Fujimura et al. (1981) developed one of the first techniques for converting the environment of the $6 / 8$ device from hydrostatic to non-hydrostatic, thus making the $6 / 8$ device a sort of deformation apparatus. By making the elastic character and/or mechanical strength of a sample assembly anisotropic, one can create a deviatoric stress in the assembly (e.g., Fig. 8a). This deviatoric stress can be relaxed by plastic flow upon heating. This technique has been used in numerous studies (Bussod et al. 1993; Durham and Rubie 1998; Fujimura 1989; Green et al. 1990; Karato et al. 1998; Karato and Rubie 1997; Weidner et al. 2001; Weidner et al. 1998). One typically achieves the anisotropic strain by embedding a rigid sample column in the compliant pressure medium. As the assembly is pressurized at room temperature (where most relevant geologic materials are very strong), a large deviatoric stress is generated on the sample. When temperature is increased, the sample softens and begins to flow, and the stress gradually relaxes. The analysis by Karato and Rubie (1997) (see also Durham and Rubie [1998]) showed that the mode of deformation in most cases is this "stress relaxation," i.e., the magnitude of deviatoric stress changes significantly within a single experiment.

This technique has several limitations. First, pressurization and plastic deformation are not completely separated; therefore, deformation during pressurization likely occurs unless special care is taken in sample assembly to absorb the initial-stage shape change of sample assembly (Durham and Rubie 1998; Karato and Rubie 1997). Second, deformation by this method is "stress relaxation," and the interpretation is complicated because of the change in stress that could cause the change in deformation mechanisms. Third, the amount of inelastic displacement is limited to the amount that can be imposed elastically during pressurization, usually well under $100 \mu \mathrm{m}$. By using a thin sample sandwiched between two pistons cut at $45^{\circ}$, Karato and Rubie (1997) were able to convert this very small displacement to relatively large strains $(\gamma \approx 1-2)$ (Fig. 8d), yet the maximum strain is not large enough to obtain steady-state fabrics. Quasi-constant displacement rate tests can also be made with multianvil apparatus through the continuous movement of the hydraulic ram (Bussod et al. 1993; Green et al. 1990), although one must realize that pressure is increasing steadily and significantly in such tests.

The multianvil deformation technique has been applied to $\sim 25 \mathrm{GPa}, \sim 2000 \mathrm{~K}$ with a sample dimension of $\sim 1-2 \mathrm{~mm}$ diameter and $\sim 2-4 \mathrm{~mm}$ long, or a thin $(\sim 0.2 \mathrm{~mm})$ disk sample with a similar diameter (Chen et al. 1998; Cordier and Rubie 2001). Sacrificing pressure for sample size, Green et al. (1990) deformed 3-mm diameter x 6-mm length samples in a $6 / 8$ device. The major advantage of this technique as compared with a diamond-anvil cell technique is that rheological properties can be investigated under more homogeneous high temperature and pressure conditions with a better-controlled 
chemical environment. Also, because relatively large samples can be used, microstructural evolution during deformation and its effects on rheology can be investigated (Green et al. 1990, 1992; Karato 1998a; Karato et al. 1998).

The limited plastic deformation is a serious shortcoming of the method. The displacement available is generally not sufficient to probe important rheological questions about lattice preferred orientation and strength in the steady state. Second, because the stress magnitude changes during an experiment, dominant mechanisms of deformation may change in a single run, making interpretation of mechanical data and microstructures difficult. This last point, however, may turn out to be an advantage for the study of rheology at a very small strain-rate (small stress) that is relevant to Earth.

\section{Diamond-anvil cell}

The highest pressures obtainable in the laboratory are in the diamond-anvil cell. This device loads the sample uniaxially and, thus, naturally produces a deviatoric stress environment. The first examinations of differential stresses in the diamond cell were motivated by the need to produce a hydrostatic environment. Use of the diamond cell as a deformation device was pioneered by Kinsland and Bassett (1977) and Sung et al. (1977). The former used X-ray transparent gaskets and passed the X-ray beam through the gaskets, perpendicular to the diamond-cell axis. Stress was measured from the shape of the Debye rings as discussed above. The measurements provided the first estimates of the room temperature strength of $\mathrm{MgO}$ at high pressures. Sung et al. (1977) developed the methodology for using pressure gradients to deduce shear stress. In this case, a sample is sandwiched between two single crystals of diamond, and radial distribution of pressure is determined by measuring the shift of fluorescence lines of ruby crystals located at various points in the sample space. Then, assuming that the pressure gradient is supported by the sample strength, one can estimate the sample's strength from the equation for force balance. This technique has been used extensively by Meade and Jeanloz (1988a,b; 1990) at room temperature to the pressure of $\sim 40 \mathrm{GPa}$.

Chai and Brown (1996) developed a diamond-cell piezometer based on the splitting of the ruby fluorescence lines. They find that the $\mathrm{R}_{2}$ line reflects the average stress, that the splitting between the $R_{1}$ and $R_{2}$ lines is sensitive to the differential stress, and that the character of the sensitivity depends on whether the load is applied parallel to the $c$-axis or the $a$-axis. This phenomenon suggests experiments that can be done with a diamond-anvil cell at room temperatures using single crystal ruby as the pressure/stress calibrant.

The major advantage of diamond cell studies is the high pressures that can be used under which a sample can be plastically deformed Wenk et al. (2000) recently deformed $\mathrm{Fe}$ at $\sim 220 \mathrm{GPa}$ ). However, there are major limitations with this approach: homogeneous heating is difficult in a diamond-anvil cell, and almost all previous results were obtained at room temperature. In addition, the strain rate is not well constrained even though deformation is likely to be time-dependent in these tests. Furthermore, the sample space is so small that some important effects such as grain-size sensitivity of strength are difficult to measure.

Poirier et al. (1981) and Sotin and Poirier (1990) report on a sapphire cell that enables larger sample volumes than a diamond cell and is more versatile for higher temperature studies. They calibrate the stress field from the characteristics of the loading system and observe the movement of strain markers in the sample. This enables them to define strain rate along with the stress determinations.

\section{Deformation-DIA}

Samples of $\mathrm{MgO}$ and polycrystalline tantalum were deformed in the deformationDIA (D-DIA) at the National Synchrotron Light Source (NSLS) in February 2002. This 
marked the first materials test ever above $P=10 \mathrm{GPa}$ in which virtually all relevant rheological independent variables were under full and independent operator control. We provide here a detailed description of this new apparatus.

The D-DIA (Wang et al. 2002) is a modification of the cubic anvil device known as the DIA (e.g., Osugi et al. (1964) and Shimomura et al. (1985)) a single-stage, wedged guide block machine. The hydrostatic DIA usually operates to $\sim 15 \mathrm{GPa}$ and $\sim 2000 \mathrm{~K}$. The D-DIA modification gives independent displacement control to one pair of opposing anvils, reducing the cubic symmetry of the DIA to tetragonal, thus allowing high-strain deformation experiments to $15 \mathrm{GPa}$.

The original DIA consists of symmetric upper and lower guide blocks, four wedge-shaped thrust blocks, and six anvils, as indicated in Figure 9. Four of the anvils are mounted on the inside faces of the thrust blocks and the other two are mounted on the inside central faces of the guide blocks, the square fronts of the anvils thus defining a cubic volume at the center of the apparatus. The operation of the DIA can be visualized by recognizing that the eight inner inclined surfaces of the guide blocks define a virtual regular octahedron whose dimension changes with the separation distance between the guide blocks. The six anvils are aligned with the six apices of this virtual octahedron, and parts are machined such that the (fixed) distance from anvil face to associated apex of the virtual octahedron is precisely the same for each anvil. As the guide blocks close or open, all displacements of the anvils are symmetric about the center. The force of a single hydraulic actuator (or ram) applied along the vertical axis in Figure 9 closes the guide blocks and, thus, compresses the cell hydro-

Figure 9. The original hydrostatic DIA apparatus. A single hydraulic ram (large arrows) drives 2 wedgetype guide blocks toward one another. The 6 anvils (truncated pyramids on the guide blocks and wedge blocks; only 5 are visible here) are thus driven toward one another, symmetrically compressing a cubic sample assembly (not shown) at the very center. statically. Ram forces of $100 \mathrm{~T}$ are typically required with various truncation sizes (typically 3 to $6 \mathrm{~mm}$ ) to reach maximum pressures near $20 \mathrm{GPa}$ (see e.g., Shimomura et al. (1992) and Utsumi et al. (1992)). By the cubic symmetry of the DIA assemby, this force is divided into three equal portions along each of the three orthogonal directions defined by opposing pairs of anvils.

Independent control of one anvil pair in D-DIA is provided by two additional hydraulic actuators - called differential rams - within each guide block (Fig. 10). These rams may be thought of not as parts that are added, but rather as a central portion of each guide block that has been cut free so that it can move vertically. A small hole is introduced into the side of each guide block to allow access for hydraulic fluid to each ram, and seals for the pressurized plenum below each differential ram are provided. The 


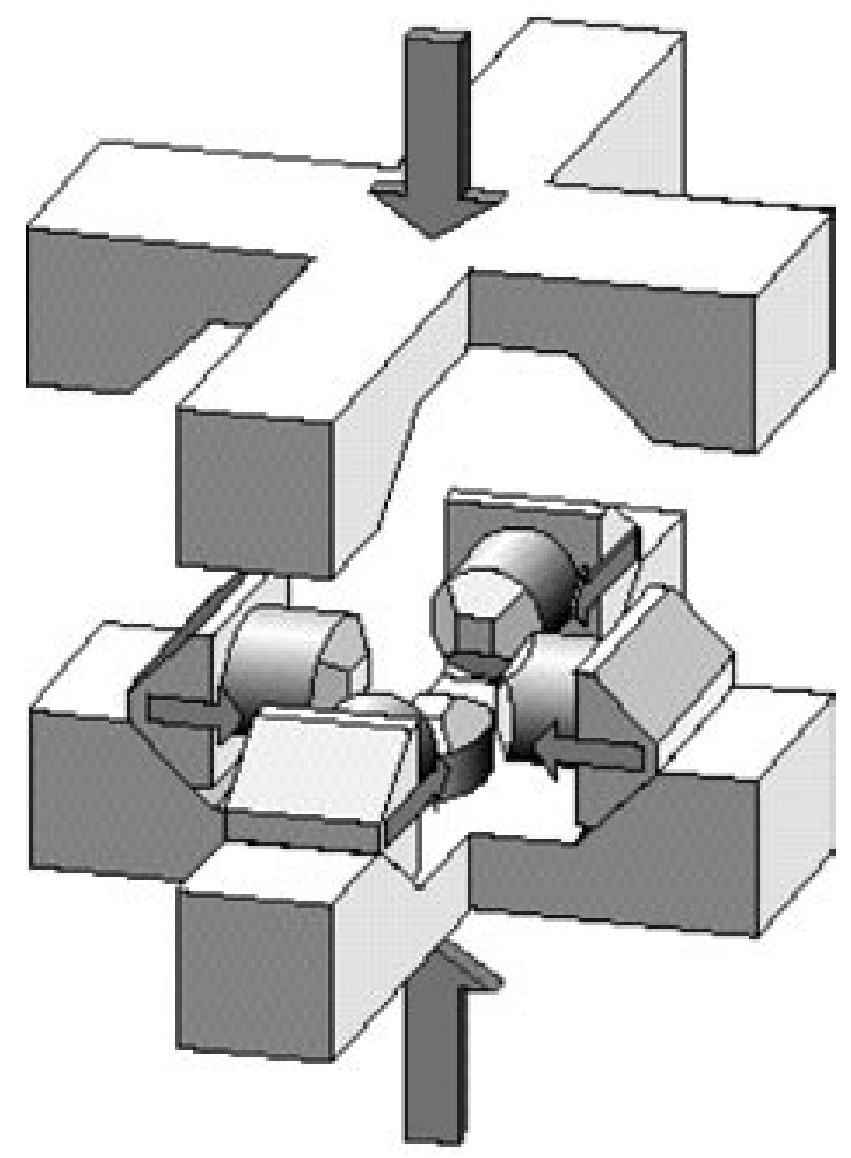

Figure 9 
conical shape of the differential rams is rather unimportant; it leaves more steel on the guide blocks and makes them slightly stronger. Note that in the D-DIA modification, the guide blocks not only support the forces confining the thrust blocks, they now have also become thick-wall pressure containers for the hydraulic fluid driving the differential rams. For this reason, the guide blocks of the D-DIA are complete discs rather than crossed steel members as in the original DIA (Fig. 9).

Figure 10. Computer-generated $3 / 4$ section through the D-DIA. Comparing to Figure 9, the guide blocks are now full cylindrical shapes, but the four wedge blocks and six anvils are identical to those in Figure 9. The two differential rams, the large conical pieces within the top and bottom guide blocks, move independently of the guide blocks themselves, and can thus generate a deviatoric state of stress in the assembly at the center (not shown). In actual operation, as the differential rams displace inward toward the center, the guide blocks move apart to allow the four wedge blocks to displace outward. The displacement rates are independently controllable and are usually chosen such that the net volume of the sample assembly, and therefore the confining pressure, remains constant.

The differential rams have a (pressurized) diameter of $89 \mathrm{~mm}$ and are therefore capable of generating a force of $125 \mathrm{~T}$ at a hydraulic pressure of $0.2 \mathrm{GPa}$, sufficient to overcome the force of the main ram (up to $33 \%$ of the main ram maximum of $300 \mathrm{~T}$ ) and apply differential force even at the highest confining pressure. Finite element analysis of the D-DIA guide blocks show that the maximum octahedral stress occurs on the inner bore in a "typical" operation where the differential load is $67 \%$ of the main ram load (presuming some force is needed to overcome packing friction and sample strength) (Wang et al. 2002). The stress at the base of the inclined surface of the guide block, the next highest point of stress concentration in the guide block, is roughly $90 \%$ of the stress in the bore. Note that in the original DIA with very large notches cut in the guide blocks, the stress concentration at the base of the inclined surfaces is twice that of the D-DIA under a similar main ram load.

The key point of independent control of the differential rams is that deformation can be imposed without the necessity of increasing confining pressure (which has been the 


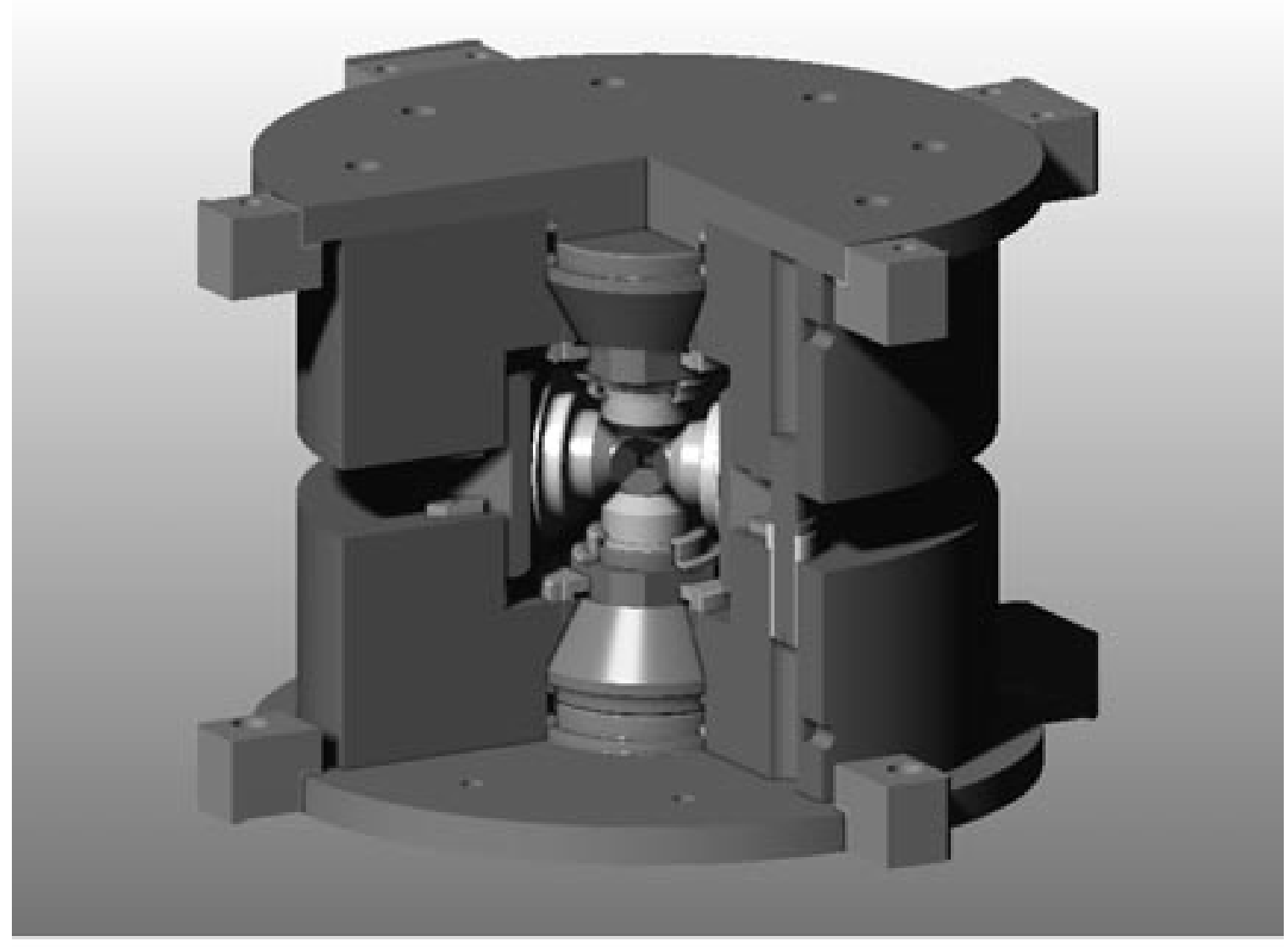

Figure 10 
long-standing limitation of deformation studies in 6/8 multianvil and diamond-anvil assemblies). In normal operation, the D-DIA sample is brought to run conditions of $P$ and $T$ with differential rams fully withdrawn in the same manner as the hydrostatic DIA. Advancing the differential rams then introduces a non-cubic shape change to the assembly. Furthermore, by simultaneously draining hydraulic fluid from the main ram at an appropriate rate, the four side anvils retract, and the total force of the main ram as well as the volume of the sample cell can be held constant. In the synchrotron X-ray beamline) pressure itself can be monitored and, accordingly, serves as the process variable. The operation at NSLS in February 2002, showed that this procedure gave very satisfactory control of pressure over very large displacements of the differential rams (Fig. 11).

The differential rams are driven by high-precision syringe pumps, and their velocities are controllable from approximately $10^{-7}$ to $10^{-2} \mathrm{~mm} / \mathrm{s}$. When both rams are driven symmetrically, this translates to a strain rate on a typical $1-\mathrm{mm}$-length, $1-\mathrm{mm}^{3}$ volume sample of $2 \times 10^{-2} \geq \dot{\varepsilon} \geq 2 \times 10^{-7} \mathrm{~s}^{-1}$. These rates are much faster than most relevant rates in geology, but as with all experimental rock mechanics work-for which these rates are typical - it is the human time scale and not the geologic time scale that governs the duration of an experiment. As with most experimental studies of rock deformation, appropriate scaling analysis is critical to apply these results to Earth. Differential ram displacements of $>1 \mathrm{~mm}$ are possible, so strains in pure shear compression can approach 1 . Deformation in simple shear, using $45^{\circ}$-cut pistons (see "Sample Assemblies," above) is also possible, allowing for much higher strains, at some cost to sample volume. Finally, the D-DIA is capable of extensional, as well compressional, deformation, because the sense of motion of the six anvils can be reversed.

\section{Rotational Drickamer apparatus (RDA)}

Large strain deformation experiments can be conducted in the torsion mode, allowing a detailed study of deformation microstructures as well as rheology. Paterson and Olgaard (2000) described such an apparatus in which a torsion actuator is attached to a gas-medium high-pressure apparatus. This apparatus contains an internal load-cell, making possible a precise measurement of stress. However, the maximum pressure of operation is limited to $\sim 0.5 \mathrm{GPa}$. Deformation experiments can be conducted at higher pressures by twisting a thin sample between anvils. Bridgman (1935a; 1937) was the first to apply the technique to $5 \mathrm{GPa}$ with an apparatus that consisted of two fixed anvils bearing on a flat, rotating anvil. The design was later modified by Griggs et al. (1960) to remove the intermediate anvil and rotate one of the anvils instead. Similar attempts were made in the late 1960s at room temperatures and at pressures to $7 \mathrm{GPa}$ (Abey and Stromberg 1969; Riecker and Seifert 1964) in which a sample was sheared, and the strength was determined by the measurements of torque on an anvil needed to deform the sample.

Figure 11 (next page). Results plotted vs. run time from a D-DIA experiment on a stacked sample of tantalum (starting length $1 \mathrm{~mm}$ ) and $\mathrm{MgO}$ (starting length $1.5 \mathrm{~mm}$ ), both polycrystalline. (a) Five traces showing, from top to bottom: measured hydraulic oil pressure in the top and bottom differential rams (scale on right-hand axis); combined centerward displacement of the two rams as measured by displacement transducers outside the sample assembly; shortening of the MgO portion of the sample; and shortening of the Ta component (scale for last three on left-hand axis). Sample length vs. time was measured directly using X-radiography. (b) Detail of the sample shortening, also indicating the strain rates (displacement rates normalized by sample length). The run was conducted by pumping hydraulic fluid to the differential rams at two different constant rate (hence deformation steps (1) and (2)), while draining fluid from the main ram in order to keep pressure at a constant level of $10 \pm 1 \mathrm{GPa}$. Note that shortening rates of the samples were also approximately constant. The steady increase in differential ram pressure during both deformation steps is the result of steadily increasing friction on the gaskets squeezed between the differential and side anvils. 


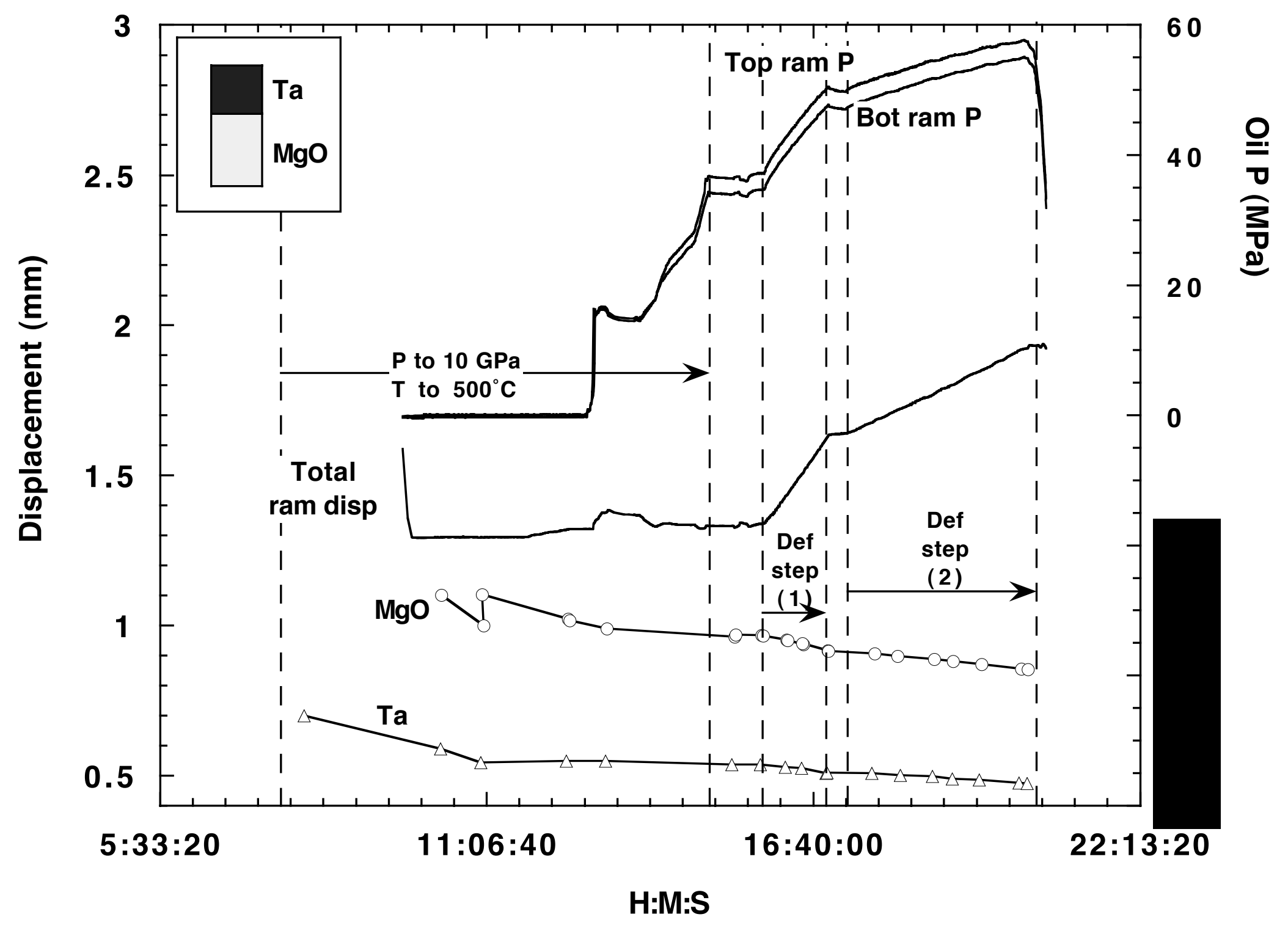




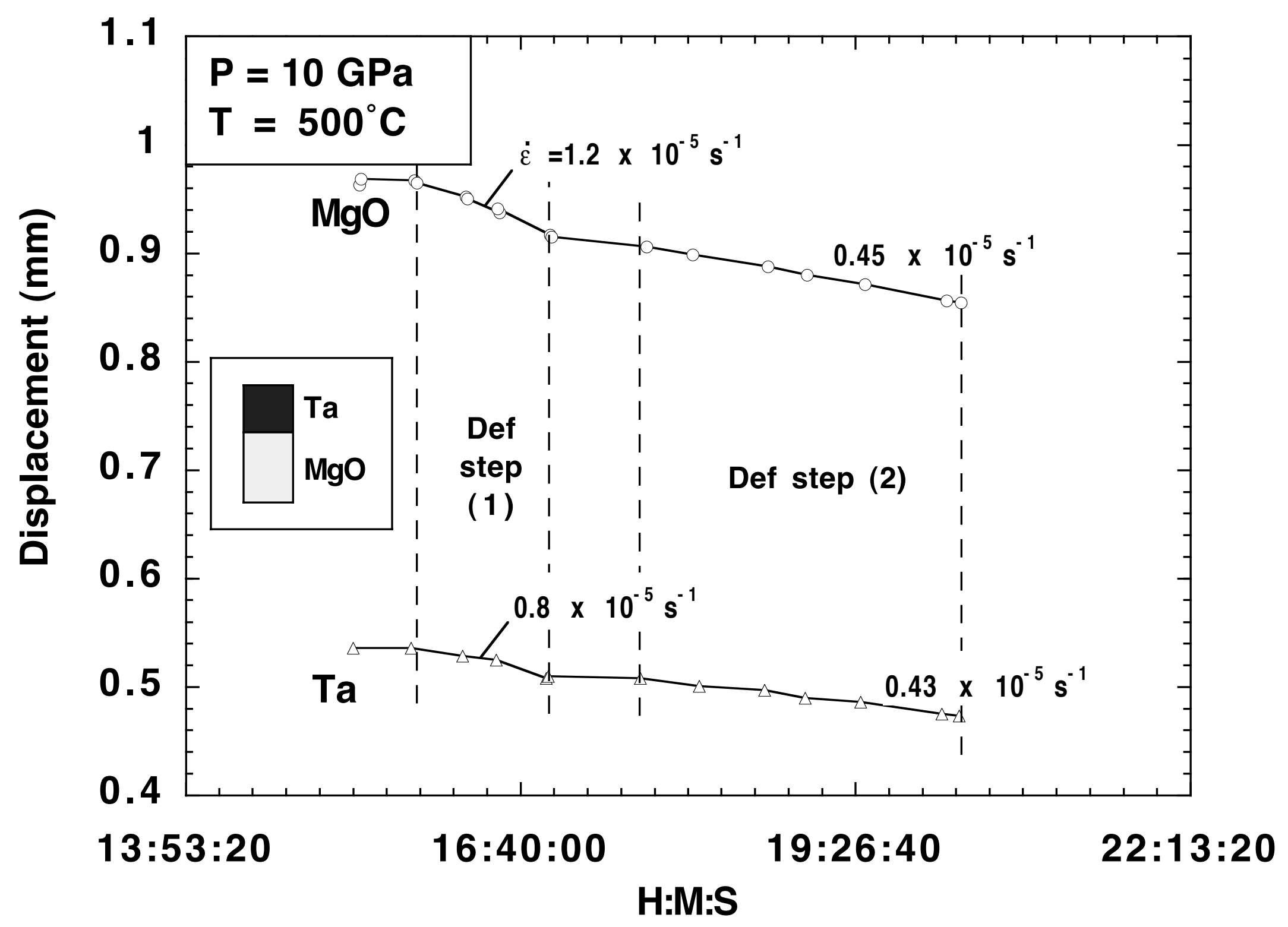


Yamazaki and Karato (2001) have improved these techniques by modifying the Drickamer-type high-pressure apparatus (Fig. 12). This apparatus allows large rotational shear deformation of a sample at higher pressures and temperatures. A Drickamer apparatus consists of a pair of anvils in a cylinder with a gasket material between the anvils and a thin disk of sample squeezed between the anvils to reach high pressure 
Figure 12. Sketch of a rotational Drickamer apparatus (Yamazaki and Karato 2001).

Figure 13. Sample assembly for a rotational Drickamer apparatus. A thin disk of sample is sandwiched between two anvils. A sample is cut into two pieces and $\mathrm{W} 5 \% \mathrm{Re}$ and $\mathrm{W} 25 \% \mathrm{Re}$ foils are inserted vertically; these act both as strain markers and as a thermocouple. The change in shape of these foils can be measured after an experiment or during an experiment through X-ray absorption. A small cylindrical W3\%Re at the center acts as an electrode for the heater and as one component of the thermocouple with a $\mathrm{W} 25 \% \operatorname{Re}$ foil.

(Fig. 13). The gasket provides an extra support for the anvils and, as a result, this apparatus yields significantly higher pressures than does a Bridgman apparatus without a gasket (Perez-Albuerne et al. 1964; Prins 1984). Because much of the force is supported by the gasket and the sample itself, the cylinder does not support a large load. Consequently, it is possible to make holes in the cylinder to provide a path for X-rays; the 


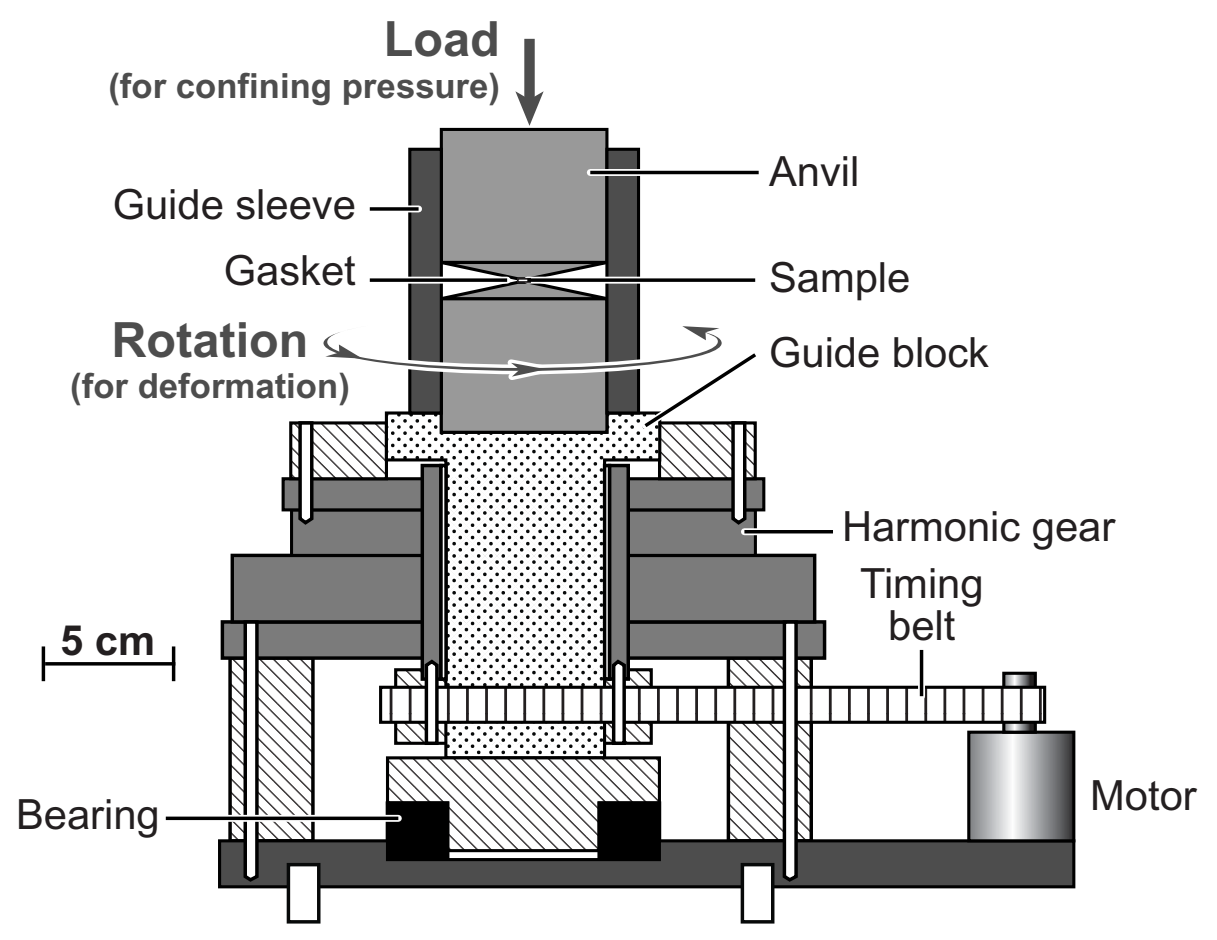



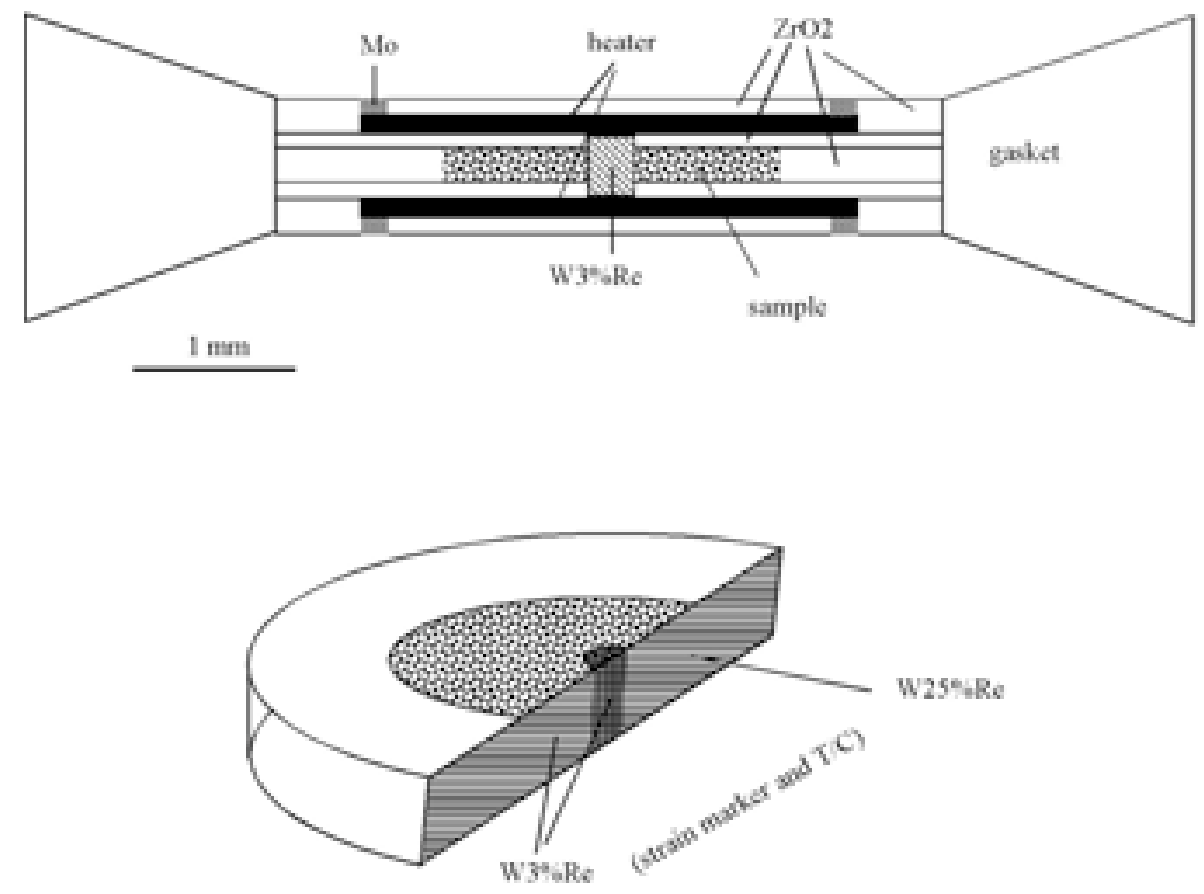

Figure 13 
thermocouple leads can be taken through these holes. The X-ray path feature is important for high-resolution stress and temperature measurements, both of which are critical for rheological studies. The Drickamer apparatus has recently been used for the in situ measurements of static properties using a synchrotron X-ray facility under conditions up to $\sim 35 \mathrm{GPa}$ and $\sim 2000 \mathrm{~K}$ (Funamori and Yagi 1993).

In the Rotational Drickamer Apparatus (RDA) modification, one of the anvils is fixed with the frame, whereas another is attached to a rotational actuator. A gearbox with an ac-servo motor provides controlled rotation of one of the anvils. A sample is first pressurized (and heated), and then the rotational actuator is started. The sample is twisted between the two anvils, and the geometry of deformation is rotational shear. The strain (and strain rate) increases from zero to the maximum value, which is determined by the motor rotation speed and sample thickness. With the current motor and gear combination, a shear strain rate from $\sim 0$ to $\sim 10^{-3} \mathrm{~s}^{-1}$ can be realized. This design has three advantages. First, the motion of the anvil for deformation is orthogonal to the motion of the anvil for pressurization. Therefore, pressurization and deformation can be clearly separated. Second, unlike axisymmetric deformation, the shape of the sample does not change appreciably during rotational deformation, allowing very large strain deformation and making possible studies of microstructural evolution. Third, because of the radial gradient in strain (strain rate), microstructural evolution and/or rheology at different strain-rates can be investigated in a single run.

The diameter of the anvil tip in the current RDA is $4 \mathrm{~mm}$ (sample diameter is $\sim 1-2$ $\mathrm{mm}$, thickness $\sim 0.2-0.4 \mathrm{~mm}$ ). With a load of $\sim 30 \mathrm{~T}$, a pressure of $\sim 15 \mathrm{GPa}$ can be generated using tungsten carbide anvils. The maximum pressure is determined primarily by the strength of anvil materials (and the diameter of sample space) and is expected to be more than $\sim 30 \mathrm{GPa}$ when a harder material such as sintered diamond or cubic-BN is used. The rotational Drickamer apparatus constructed at Yale University has been tested at $\mathrm{P}=12 \mathrm{GPa}, \mathrm{T}=1473 \mathrm{~K}$. A polycrystalline sample of $\mathrm{MgO}$ was deformed homogeneously to the maximum shear strain of $\sim 3$. Two methods can be employed to estimate the stress magnitude. First, X-ray diffraction techniques can be used for deformation experiments with the RDA in a synchrotron radiation facility in the manner discussed previously. At this writing, such experiments are planned but have not yet been carried out. To adopt the diffraction technique for rotation geometry, a conical shape window is made in a cylinder to collect diffracted X-rays for a range of angles (Fig. 14b). One complication with this apparatus is the radial variation of stress. This is inevitable in the torsion test (e.g., Paterson and Olgaard 2000); Fig. 14a). In measuring the stress magnitude by X-ray diffraction, one must consider the spatial resolution. Important factors that control the spatial resolution are the cross-sectional area of the X-ray beam and the $2 \theta$ value used for diffraction (with energy dispersive mode). A smaller beam size and a larger $2 \theta$ are preferred for a better spatial resolution of stress measurements. However, a smaller beam size results in a weaker signal. Also the $2 \theta$ values are limited by the energy of X-ray and the $d$-spacings as well as the geometry of the apparatus (we will use $\sim 10-50 \times 10-50 \mu \mathrm{m}^{2}$ beam [slit] size and $2 \theta \approx 8-10^{\circ}$ ). To allow for control of $2 \theta$, the apparatus is set on a mobile stage (with a remote control through a stepping motor) whose angle and position with respect to the beam line can be adjusted to optimize the conditions. The resolution of the stress measurement is also dependent on the detector. We anticipate that a spatial resolution of $100-200 \mu \mathrm{m}$ and the stress resolution better than $0.1 \mathrm{GPa}$ will be obtained by this technique. The use of a hollow specimen could help solve the problem of stress heterogeneity (Fig. 14d).

We note that this technique not only allows us to estimate the stress magnitude, but also provides information as to the grain-scale stress (strain) distribution in a polycrystalline material (either homogeneous stress or homogeneous strain (Funamori et 
Figure 14. (A) Stress distribution in a sample in a torsion test ( $\mathrm{n}$ is stress exponent, $\ddot{\gamma}=\mathrm{A} \tau^{\mathrm{n}}$ ). (B) X-ray diffraction geometry. Stress magnitude can be estimated from the difference in $d$-spacing collected from two different diffracted beams through two windows that are cut at $45^{\circ}$ with respect to the vertical axis. (C) A top view of a sample and the X-ray path. In order to determine the stress, $\mathrm{X}$-ray diffraction must occur in a region of a sample where the shear direction is normal to the X-ray beam. (D) The geometry of a hollow sample, where stress heterogeneity is less.

al. 1994). Such information is useful in understanding the physics of deformation of polycrystals (Kocks 1970).

Another method is to use dislocation density as a stress indicator. This technique can be used when the deformation mechanism is dislocation creep and when stress has been held at a constant value for at least a few percent strain. The method does not work well if stress is inhomogeneous. We have recently used this technique for olivine and are able to estimate the stress magnitude with an uncertainty of $\sim 10-15 \%$ (Jung and Karato 2001; Karato and Jung 2002 in press). The reliability of such measurements for high-pressure phases is not as high as that for olivine because the calibration curve for dislocation density vs. stress relationship is not available. However, the relation between dislocation density and stress is nearly universal,

$$
\rho=\alpha b^{-2}(\sigma / \mu)^{2}
$$

( $\rho$ : dislocation density, $\alpha$ : a constant of order unity, $b$ : the length of the Burgers vector, $\sigma$ : stress, $\mu$ : shear modulus (e.g., Kohlstedt and Weathers 1980) We consider that such measurements should provide at least semi-quantitative data on the stress magnitude.

We will perform both single-layer deformation experiments and two-layer deformation experiments. A single-layer deformation experiment is simpler to interpret, but the big advantage of a two-layer design is that one can determine the relative strength of two samples very accurately. Our experience with this apparatus showed that we can deform our samples to strains up to at least $\gamma \approx 10$ at $\sim 15 \mathrm{GPa}$. Such a large strain is essential for the study of deformation fabric.

\section{SUMMARY AND PERSPECTIVES}

We have summarized some of the recent developments in quantitative characterization of stress and strain and the controlled generation of deviatoric stresses under high pressure (and temperature). These developments will allow us to explore 
(A)

(B)

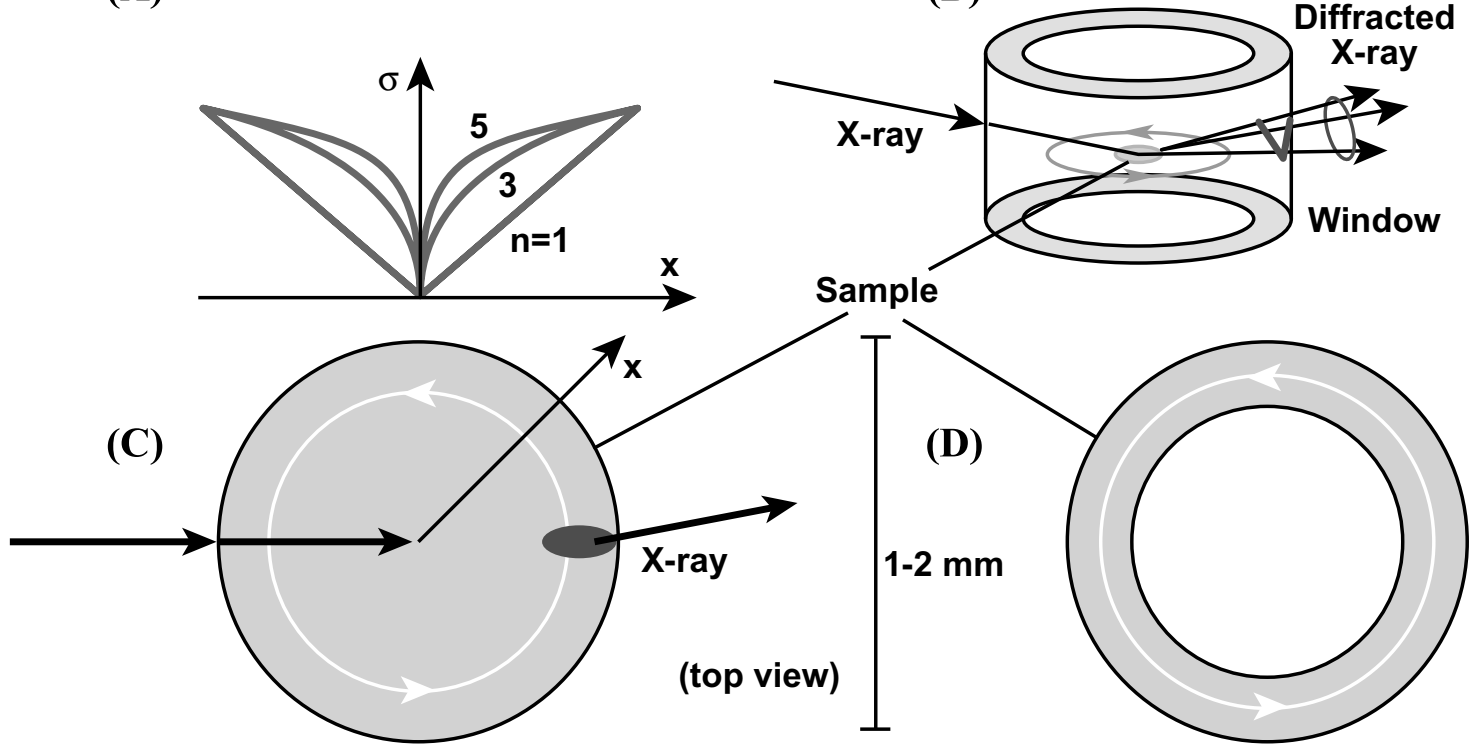

Figure 14 
rheological behavior of Earth materials under much deeper conditions than heretofore possible. Such studies will, for the first time, provide the critical data sets necessary for understanding whole mantle dynamics through modeling and/or through the analyses of observations such as seismic anisotropy. Further technical developments need to be made to make these techniques robust. First, stress measurements using different techniques must be benchmarked. Three techniques are currently used to measure stress under high pressure: (1) Measurement of force using an external load-cell with a low-friction sample design (Tingle et al. 1993), (2) synchrotron X-ray measurement of orientation dependence of $d$ spacing (Singh et al. 1998), and (3) inference from dislocation densities (Karato and Jung 2002 in press). Below $\sim 4 \mathrm{GPa}$, all of these techniques can be employed to determine the strength of a standard materials such as olivine. A comparison of results of these measurements will provide a measure of reliability of each technique. Second, a number of issues still remain regarding the sample assembly. They include a proper control of thermodynamic environment, particularly water fugacity (or water content) and oxygen fugacity. Third, in almost all of these techniques under high pressure, it is almost inevitable to have deformation during pressurization. An experimental procedure (e.g., annealing; a very low strength medium surrounding the sample) must be established to minimize the effects of initial stage deformation.

It must also be emphasized that the sample preparation and characterization are as critical as careful mechanical tests. Samples with negligible porosity must be used and grain size and water content must be measured both before and after each experiment (control of other thermodynamic variables such as oxygen fugacity is also important; see Rubie et al. (1993)). In almost all laboratory deformation experiments, conditions are often close to the boundary between dislocation and diffusion creep (Karato et al. 1998), and, therefore, precise measurements of grain size are needed to interpret the data. Also, water content must be measured both before and after each experiment. Water is known to have significant effects on rheology, but its content in samples is difficult to control during high-pressure experiments.

Using the results of mechanical tests and sample characterization, dominant microscopic mechanisms of deformation must be identified. A meaningful comparison of strength of different materials (or for the same material for different conditions) can only be made when the deformation mechanism is the same, and the comparison must be made based on an appropriate scaling. For instance, if the dominant mechanism of deformation is diffusion creep, then the data must be compared by normalizing with respect to the grain size.

With the use of these new techniques (combined with careful characterization of samples and the analyses of deformation mechanisms), we will be able to cast first light on the rheology of the more than $90 \%$ of Earth's mantle that has not been accessible by quantitative studies. Results of such studies will be critical to our better understanding of dynamics and evolution of this planet.

\section{ACKNOWLEDGMENTS}

We are most grateful to H. W. Green, II, for reviewing the manuscript. Funding was provided by the National Science Foundation under award EAR-0135551. Work by WBD performed under the auspices of the U.S. Department of Energy by the Lawrence Livermore National Laboratory under contract W-7405-ENG-48. Additional portions of this work were performed at GeoSoilEnviroCARS (GSECARS), Sector 13, Advanced Photon Source at Argonne National Laboratory. GSECARS is supported by the National Science Foundation - Earth Sciences, Department of Energy - Geosciences, W.M. Keck Foundation, and the U.S. Department of Agriculture. Use of the Advanced Photon Source 
was supported by the U.S. Department of Energy, Basic Energy Sciences, Office of Energy Research, under Contract No. W-31-109-Eng-38.

\section{REFERENCES}

Abey AE, Stromberg HD (1969) 70 kilobar shear apparatus. Rev Sci Instrum 40:557

Borch RS, Green HW II (1989) Deformation of peridotite at high pressure in a new molten cell: Comparison of traditional and homologous temperature treatments. Phys Earth Planet Inter 55: 269-276

Boyd FR, England JL (1960) Apparatus for phase equilibrium measurements at pressures up to 50 kilobars and temperatures up to $1750^{\circ} \mathrm{C}$. J Geophys Res 65:741-748

Bridgman PW (1914) The technique of high pressure experimenting. Proc Am Acad Arts Sci 49:627-643

Bridgman PW (1935a) Effects of high shearing stress combined with high hydrostatic pressure. Phys Rev 48:825-847

Bridgman PW (1935b) Polymorphism, principally of the elements, up to 50,000 kg/cm ${ }^{2}$. Phys Rev 48: 893-906

Bridgman PW (1937) Shearing phenomena at high pressures, particularly in inorganic compounds. Proc Am Acad Arts Sci 72:45-136

Bridgman PW (1942) Pressure-volume relations for seventeen elements to $100,000 \mathrm{~kg} / \mathrm{cm}^{2}$. Proc Am Acad Arts Sci 74:425-440

Bridgman PW (1948) The compression of 39 substances to 100,000 kg/cm². Proc Am Acad Arts Sci 76: 55-70

Bridgman PW (1952a) The resistance of 72 elements, alloys, and compounds to 100,000 kg/ $\mathrm{cm}^{2}$. Proc Am Acad Arts Sci 81:65-NN

Bridgman PW (1952b) The Physics of High Pressure. G Bell and Sons, London

Bridgman PW (1952c) Studies in Large Plastic Flow and Fracture with Special Emphasis on the Effects of Hydrostatic Pressure. McGraw-Hill, New York

Burnley PC, Green HW II, Prior D (1991) Faulting associated with the olivine to spinel transformation in $\mathrm{Mg}_{2} \mathrm{GeO}_{4}$ and its implications for deep-focus earthquakes. J Geophys Res 96:425-443

Bussod GY, Katsura T, Rubie DC (1993) The large volume multi-anvil press as a high P-T deformation apparatus. Pure Appl Geophys 141:579-599

Carter N.L, Christie J.M, Griggs D. T (1964) Experimental deformation and recrystallization of quartz. J Geol 72:687-733

Chai M, Brown JM (1996) Effects of static non-hydrostatic stress on the R lines of ruby single crystals. Geophys Res Lett 23:3539-3542

Chen J, Inoue T, Weidner DJ, Wu Y, Vaughan MT (1998) Strength and water weakening of mantle minerals, olivine, wadsleyite and ringwoodite. Geophys Res Lett 25:575-578

Cordier P, Rubie DC (2001) Plastic deformation of minerals under extreme pressure using a multi-anvil apparatus. Mater Sci Eng A309-310:38-43

Davies GF (1995) Penetration of plates and plumes through the mantle transition zone. Earth Planet Sci Lett 133, 507-516

Durham WB, Rubie DC (1998) Can the multianvil apparatus really be used for high-pressure deformation experiments? In Properties of Earth and Planetary Materials at High Pressure and Temperature, Geophysical Monograph 101. M Manghnani, Y Yagi (eds) p 63-70. American Geophysical Union

Eremets M. I (1996) High Pressure Experimental Methods. Oxford University Press

Fujimura A (1989) Preferred orientation of mantle minerals. In Rheology of Solids and of the Earth. S-i Karato, M Toriumi (eds) p 263-283. Oxford University Press

Fujimura A, Endo S, Kato M, Kumazawa M (1981) Preferred orientation of $\beta-\mathrm{Mn}_{2} \mathrm{GeO}_{4}$. In Programme and Abstracts, The Japan Seismological Society, p 185

Fukao Y, Obayashi M, Inoue H, Nenbai M (1992) Subducting albs stagnant in the mantle transition zone. J Geophys Res 97:4809-4822

Funamori N, Yagi T (1993) High pressure and high temperature in situ X-ray observation of $\mathrm{MgSiO}_{3}$ perovskite under lower mantle conditions. Geophys Res Lett 20:387-390

Funamori N, Yagi T, Uchida T (1994) Deviatoric stress measurement under uniaxial compression by a powder X-ray diffraction method. J Appl Phys 75:4327-4331

Getting IC (1998) New determination of the bismuth I-II equilibrium pressure: A proposed modification to the practical pressure scale. Metrologia 35:119-132

Getting IC, Spetzler HA (1993) Gas-charged piston-cylinder apparatus for pressures to 4 GPa. In HighPressure Science and Technology. SC Schmidt, JW Shaner, GA Samara, M Ross (eds) p 1581-1584. American Institute of Physics 
Green HW II (2001) Earthquakes at depth and their enabling mineral reactions. Eleventh Annual V.M. Goldschmidt Conference (abstr)

Green HW II, Borch RS (1989) A new molten salt cell for precision stress measurement at high pressure. European J Mineral 1:213-219

Green HW II, Houston H (1995) The mechanics of deep earthquakes. Ann Rev Earth Planet Sci 23:169-213

Green HW II, Scholz CH, Tingle TN, Young TE, Koczynski TA (1992) Acoustic emissions produced by anticrack faulting during the olivine-spinel transformation. Geophys Res Lett 19:789-792

Green HW II, Young TE, Walker D, Scholz CH (1990) Anticrack-associated faulting at very high pressure in natural olivine. Nature 348:720-722

Griggs DT (1936) Deformation of rocks under high confining pressure. J Geol 44:541-577

Griggs DT (1967) Hydrolytic weakening of quartz and other silicates. Geophys J R Astron Soc 14:19-31

Griggs DT, Turner FJ, Heard HC (1960) Deformation of rocks at $500^{\circ}$ to $800^{\circ} \mathrm{C}$. In Rock Deformation, Geol Soc Am Memoir 79. DT Griggs, JW Handin (eds) p 39-104. Geol Soc Am, Boulder, Colorado

Hager BH (1984) Subducted slabs and the geoid: constraints on mantle rheology and flow. J Geophys Res 89:6003-6015

Hall HT (1958) Some high-pressure, high-temperature apparatus design considerations: Equipment for use at 100,000 atmospheres and $3000^{\circ} \mathrm{C}$. Rev Sci Instrum 29:267-275

Handin JW (1953) An application of high pressure in geophysics: experimental rock deformation. Am Soc Mech Eng Trans 75:315-325

Heard HC, Carter NL (1968) Experimentally induced "natural" intergranular flow in quartz and quartzite. Am J Sci 266:1-42

Hill R (1950) The Mathematical Theory of Plasticity. Oxford University Press

Hirth G, Kohlstedt DL (1996) Water in the oceanic upper mantle; implications for rheology, melt extraction and the evolution of the lithosphere. Earth Planet Sci Lett 144:93-108

Hobbs BE, Ord A (1988) Plastic instabilities: implications for the origin of intermediate and deep focus earthquakes. J Geophys Res 89:10521-10540

Honda S, Yuen DA, Balachandar S, Reuteler D (1993) Three-dimensional instabilities of mantle convection with multiple phase transitions. Science 259:1308-1311

Houck JC, Hutton UO (1963) Correlation of factors influencing the pressures generated in multi-anvil devices. In High-Pressure Measurement. AA Giardini, EC Lloyd (eds). Butterworths, London

Jaeger JC, Cook NGW (1976) Fundamentals of Rock Mechanics, 2nd edition. Chapman and Hall

Jiao W, Siliver PG, Fei Y, Prewitt CT (2000) Do deep earthquakes occur on preexisting weak zones? An examination of the Tonga subduction zone. J Geophys Res 105:28125-28138

Jung H, Karato S (2001) Effect of water on the size of dynamically recrystallized grains in olivine. J Struct Geol 23:1337-1344

Karato S (1998) Micro-physics of post-glacial rebound. In Ice Age Dynamics. P Wu (ed) p 351-364. Trans Tech. ???

Karato S (1998a) Effects of pressure on plastic deformation of polycrystalline solids: some geological applications. In High Pressure Research in Materials Sciences. PY Yu (ed) p 3-14. Materials Research Society

Karato S, Dupas-Bruzek C, Rubie DC (1998) Plastic deformation of silicate spinel under the transition zone conditions of the Earth. Nature 395:266-269

Karato S, Jung H (2002 in press) Effects of pressure on high-temperature dislocation creep in olivine polycrystals. Phil Mag (in press)

Karato S, Riedel MR, Yuen DA (2001) Rheological structure and deformation of subducted slabs in the mantle transition zone: implications for mantle circulation and deep earthquakes. Phys Earth Planet Inter 127:83-108

Karato S, Rubie DC (1997) Toward experimental study of plastic deformation under deep mantle conditions: a new multianvil sample assembly for deformation studies under high pressures and temperatures. J Geophys Res 102:20111-20122

Karato S-i, Wu P (1993) Rheology of the upper mantle; a synthesis. Science 260:771-778

Kawai N, Endo S (1970) The generation of ultrahigh hydrostatic pressure by a split sphere apparatus. Rev Sci Instrum 41:1178-1181

Kellogg LH, Hager BH, van der Hilst RD (1999) Compositional stratification in the deep mantle. Science 283:1881-1884

King SD (1995) Radial models of mantle viscosity: results from a generic algorithm. Geophys J Intl 122:725-734

Kinsland GL, Bassett W (1977) Strength of $\mathrm{MgO}$ and $\mathrm{NaCl}$ polycrystals to confining pressures of $250 \mathrm{kbar}$ at 25 C. J Appl Phys 48:978-985

Kirby SH, Stein S, Okal EA, Rubie DC (1996) Metastable mantle phase transformations and deep earthquakes in subducting oceanic lithosphere. Rev Geophys 34:261-306 
Kocks UF (1970) The relation between polycrystal deformation and single-crystal deformation. Metall Trans 1:1121-1143

Kohlstedt DL, Chopra PN (1994) Influence of basaltic melt on the creep of polycrystalline olivine under hydrous conditions. In Magmatic Systems. Intl Geophysics Series, Vol. 57. MP Ryan (ed) p 37-53

Kohlstedt DL, Weathers MS (1980) Deformation-induced microstructures, paleopiezometers, and differential stresses in deeply eroded fault zones. J Geophys Res 85:6269-6285

Liebermann RC, Wang Y (1992) Characterization of sample environment in a uniaxial split-sphere apparatus. In High-Pressure Research: Application to Earth and Planetary Sciences. Y Syono, MH Manghnani (eds) p 19-31. American Geophysical Union, Washington, DC

Lloyd EC, Hutton UO, Johnson DP (1959) Compact multi-anvil wedge-type high pressure apparatus. J Res Nat Bur Stand 59:63C

Masters G, Laske G, Bolton H, Dziewonski AM (2000) The relative behavior of shear velocity, bulk sound speed, and compressional velocity in the mantle: Implications for chemical and thermal structure. In Earth's Deep Interior. S Karato, AM Forte, RC Liebermann, G Masters, L Stixrude (eds) p 63-87. American Geophysical Union, Washington, DC

Meade C, Jeanloz R (1988a) The yield strength of B1 sand B2 phases of NaCl. J Geophys Res 93: 3270-3274

Meade C, Jeanloz R (1988b) The yield strength of MgO to 40 GPa. J Geophys Res 93:3261-3269

Meade C, Jeanloz R (1990) The strength of mantle silicates at high pressures and room temperature: implications for the viscosity of the mantle. Nature 348:533-535

Mei S, Kohlstedt DL (2000a) Influence of water on plastic deformation of olivine aggregates, 1. Diffusion creep regime. J Geophys Res 105:21457-21469

Mei S, Kohlstedt DL (2000b) Influence of water on plastic deformation of olivine aggregates, 2. Dislocation creep regime. J Geophys Res 105:21471-21481

Mitrovioca JX, Forte AM (1997) Radial profile of mantle viscosity: results from the joint inversion of convection and postglacial rebound observables. J Geophys Res 102:2751-2769

Mitrovioca JX, Peltier WR (1991) A complete formalism for the inversion of postglacial rebound data: resolving power analysis. Geophys. J Intl 104:267-288

Ogawa M (1987) Shear instability in a viscoelastic material as the cause for deep earthquakes. J Geophys. Res 92:13801-13810

Onodera A (1987) Octahedral-anvil high-pressure devices. High Temp-High Press 19:579-609

Osugi J, Shimizu K, Inoue T, Yasunami K (1964) A compact cubic anvil high pressure apparatus. Rev Phys Chem Japan. 34:1-6

Paterson M. S (1970) A high-pressure, high-temperature apparatus for rock deformation. Intl J Rock Mech Min Sci 7:517-526

Paterson MS (1978) Experimental Rock Deformation: the Brittle Field. Springer-Verlag

Paterson MS (1990) Rock deformation experimentation. In The Brittle-Ductile Transition in Rocks: The Heard Volume. AG Duba, WB Durham, JW Handin, HF Wang (eds) p 187-194. American Geophysical Union, Washington, DC

Paterson MS, Olgaard DL (2000) Rock deformation tests to large shear strains in torsion. J Struct Geol 22:1341-1358

Peltier WR (1998) Postglacial variation in the level of the sea: implications for climate dynamics and solidEarth geophysics. Rev Geophys 36:603-689

Perez-Albuerne EA, Forgsgren KF, Drickamer HG (1964) Apparatus for X-ray measurements at very high pressure. Rev .Sci Instrum 35, 29-33

Poirier J-P, Sotin C, Peyronneau J (1981) Viscosity of high-pressure ice VI and evolution and dynamics of Ganymede. Nature 292:225-227

Post RL (1977) High-temperature creep of Mt. Burnett dunite. Tectonophysics 42:75-110

Prins JF (1984) A semiempirical description of pressure generation between Bridgman anvils. High TempHigh Press 16:657-664

Raleigh CB, Paterson MS (1965) Experimental deformation of serpentinite and its tectonic implications. J Geophys. Res 70:3965-3985

Riecker RE, Seifert KE (1964) Olivine shear strength at high pressure and room temperature. Geol Soc Am Bull 75:571-574

Rubey WW (1972) Foreword. In Flow and Fracture of Rocks, Vol. 16, Geophysical Monograph. HC Heard, IY Borg, NL Carter, CB Raleigh (eds) p $i x-x$. American Geophysical Union, Washington, DC

Rubie DC, Karato S-i, Yan H, O'Neill HSC (1993) Low differential stress and controlled chemical environment in multianvil high-pressure experiments. Phys Chem Minerals 20:315-322

Shimomura O, Utsumi W, Taniguchi T, Kikegawa T, Nagashima T (1992) A new high pressure and high temperature apparatus with sintered diamond anvils for synchrotron radiation use. In High-pressure research: Application to Earth and planetary sciences. Y Syono, MH Manghnani (eds) p 3-11. Terra Scientific Publishing, Tokyo 
Shimomura O, Yamaoka S, Yagi T, Wakatsuki M, Tsuji K, Kawamura H, Hamaya N, Fukuoga O, Aoki K, Akimoto S (1985) Multi-anvil type X-ray system for synchrotron radiation. In Solid State Physics Under Pressure. S Minomura (ed), p 351-356. Terra Scientific Publishing, Tokyo

Singh A. K, Balasingh C, Mao H.-k, Hemley R, Shu J (1998) Analysis of lattice strains measured under nonhydrostatic pressure. J Appl Phys 83:7567-7578

Sotin C, Poirier J-P (1990) The sapphire anvil cell as a creep apparatus. In The Brittle-Ductile Transition in Rocks: The Heard Volume, Vol. Geophys. Monogr. No. 56. AG Duba, WB Durham, JW Handin, HF Wang (eds) p 219-223. American Geophysical Union, Washington, DC

Sung CM, Goetze C, Mao HK (1977) Pressure distribution in the diamond anvil press and the shear strength of fayalite. Rev Sci Instrum 48:1386-1391

Tackley PJ, Stevenson DJ, Glatzmaier GA, Schubert G (1993) Effects of endothermic phase transition at $670 \mathrm{~km}$ depth in a spherical model of mantle convection in the Earth's mantle. Nature 361:699-704

Thoraval C, Richards MA (1997) The geoid constraint in global geodynamics: viscosity structure, mantle heterogeneity models and boundary conditions. Geophys J Intl 131:1-8

Tingle TN, Green HW II, Young TE, Koczynski TA (1993) Improvements to Griggs-type apparatus for mechanical testing at high pressures and temperatures. Pure Appl Geophys 141:523-543

Tullis TE, Tullis J (1986) Experimental rock deformation techniques. In Mineral and Rock Deformation: Laboratory Studies, The Paterson Volume. BE Hobbs, HC Heard (eds) p 297-324. American Geophysical Union, Washington, DC

Turner FJ, Griggs DT, Clark RH, Dixon RH (1956) Deformation of Yule marble. Part VII: Development of oriented fabrics at $300^{\circ}-500^{\circ} \mathrm{C}$. Geol Soc Am Bull 67:1259-1294

Utsumi W, Yagi T, Leinenweber K, Shimomura O, Taniguchi T (1992) High pressure and high temperature generation using sintered diamond anvils. In High-pressure research: Application to Earth and planetary sciences. Y. Syono and M. H. Manghnani), p 37-42. American Geophysical Union, Washington, DC

van der Hilst RD, Engdahl R, Spakman W, Nolet G (1991) Tomographic imaging of subducted lithosphere below northwest Pacific island arcs. Nature 353:37-43

van der Hilst RD, Karason H (1999) Compositional heterogeneity in the bottom 1000 kilometers of Earth's mantle: toward a hybrid convection model. Science 283:1885-1888

van der Hilst RD, Widiyantoro RDS, Engdahl ER (1997) Evidence for deep mantle circulation from global tomography. Nature 386:578-584

Vaughan M, Chen J, Li L, Weidner DJ, Li B (2000) Use of X-ray imaging techniques at high pressure and temperature for strain measurements. In Science and Technology of High Pressure, Proceedings of AIRAPT-17. MH Manghnani, WJ Nellis, M. F. Nicol (eds) p 1097-1098. Universities Press

Walker D (1991) Lubrication, gasketing, and precision in multianvil experiments. Am Mineral 76: 1092-1100

Walker D, Carpenter MA, Hitch CM (1990) Some simplifications to multianvil devices for high-pressure experiments. Am Mineral 75:1020-1028

Wang Y, Durham W, Getting I (2002) D-DIA: A new apparatus for high-temperature deformation at pressures up to $15 \mathrm{GPa}$. Rev Sci Instrum (submitted)

Weidner DJ (1998) Rheological studies at high pressure. Rev Mineral 3:493-524

Weidner DJ, Chen J, Xu Y, Wu Y, Vaughan MT, Li L (2001) Subduction Zone Rheology. Phys Earth Planet Inter 127:67-81

Weidner DJ, Vaughan MT, Ko J, Wang Y, Liu X, Yeganeh-Haeri A, Pacalo RE, Zhao Y (1992) Characterization of stress, pressure, and temperature in SAM85, A DIA type high pressure apparatus. In High-Pressure Research: Application to Earth and Planetary Sciences, Vol. Geophysical Monograph 67. Y Syono, MH Manghnani (eds) p 13-17. Terra Scientific Publishing Company, Tokyo, and American Geophysical Union, Washington, DC

Weidner DJ, Wang Y, Chen G, Ando J, Vaughan MT (1998) Rheology measurements at high pressure and temperature. In Properties of Earth and Planetary Materials at High Pressure and Temperature, Geophysical Monograph 101. M Manghnani, Y Yagi (eds) p 473-482. American Geophysical Union

Weidner DJ, Wang Y, Vaughan MT (1994) Strength of diamond. Science 266:419-422

Wenk H-R, Matthies S, Hemley RJ, Mao HK, Shu J (2000) The plastic deformation of iron at pressure of the Earth's inner core. Nature 405:1044-1047

Wu TC, Bassett WA (1993) Deviatoric stress in a diamond anvil cell using synchrotron radiation with two diffraction geometries. Pure Appl Geophys 141:509-519

Yamazaki D, Karato S (2001) High pressure rotational deformation apparatus to $15 \mathrm{GPa}$. Rev Sci Instrum 72:4207-4211 
contents

INTRODUCTION .1

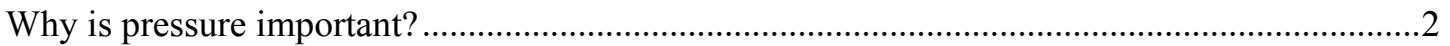

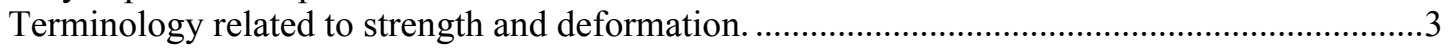

A BRIEF HISTORY OF HIGH-PRESSURE APPARATUS.......................................................

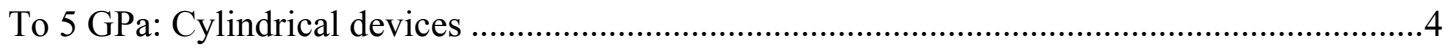

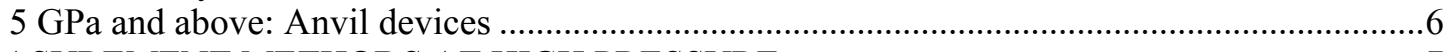

MEASUREMENT METHODS AT HIGH PRESSURE …..........................................................

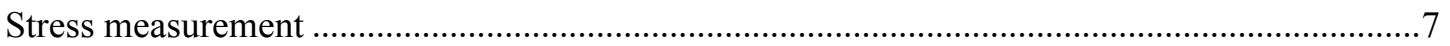

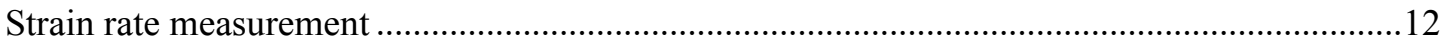

MODERN TECHNIQUES FOR DEFORMATION AT HIGH PRESSURES ..............................14

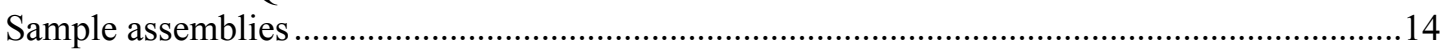

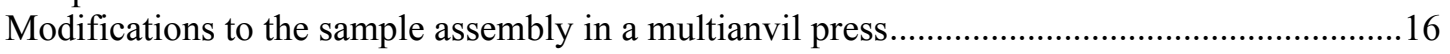

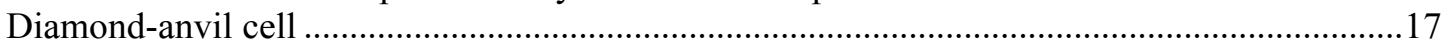

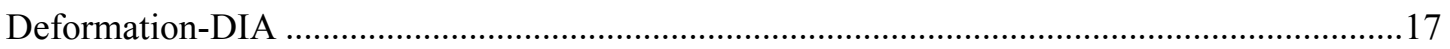

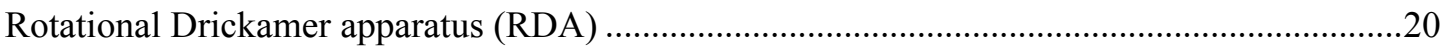

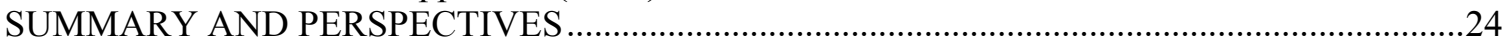

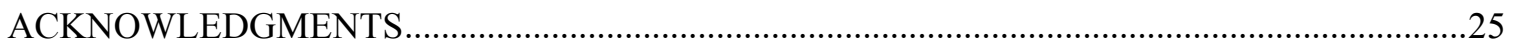

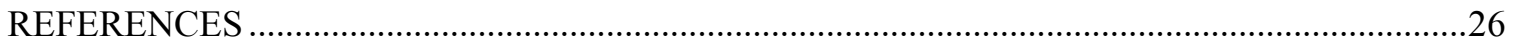

